\title{
Wenn GotT Und Teufel im Marketing tätig SIND: CHRISTLICH VS. HEIDNISCH, GÖTTLICH VS. TEUflisch in NAHRUNGS-, GENUSS- UND Heilmittelnamen. (Betrachtungen zU BenenNung UND BEZEICHNUNG VON ESS- UND TRINKBAREM IM GEGENWÄRTIGEN (FACH)SPRACHGEBRAUCH)
}

\author{
Rodica-Cristina ȚURCANU \\ Universitatea Tehnică din Cluj-Napoca, \\ Centrul Universitar Nord din Baia Mare, România
}

\begin{abstract}
When God and the Devil are involved in marketing: Christian vs. pagan, divine vs. devilish in names of basic /semi-luxury foods and remedies (Considerations on naming foods, drinks and remedies in contemporary language use and/or languages for special purposes.)
\end{abstract}

\begin{abstract}
After a short overview of the motivation behind naming foods and drinks with names in the field of religion, the paper aims at considering name-giving in the case of basic/semiluxury foods and remedies in the world of brands and consumers from the perspective of marketing. The numerous examples in several languages seek to prove that products meant for human consumption and well-being become more attractive, tempt consumers and manipulate their behavior when they bear names that refer to God, the Devil, angels, magic and the like.
\end{abstract}

Keywords: naming, commercial onomastics, religion, brand, marketing, consumer behaviour.

Nicht vom Wohlwollen des Metzgers, Brauers und Bäckers erwarten wir das was wir zum Essen brauchen, sondern davon, daßsie ihre eigenen Interessen wahrnehmen. Wir wenden uns nicht an ihre Menschen-, sondern an ihre Eigenliebe, und wir erwähnen nicht die eigenen Bedürfnisse, sondern sprechen von ihrem Vorteil. (Adam Smith, 1723-1790) ${ }^{1}$

\section{Einführung}

Im gegenwärtigen Kontext der vorherrschenden Betonung auf individuelle Interessen und materiellen Gewinn, habe ich mich gefragt, ob die Namensgebung für

1 Adam Smith An Inquiry into the Nature and Causes of the Wealth of Nations I.II.2; 17 It is not from the benevolence of the butcher, the brewer, or the baker, that we expect our dinner, but from their regard to their own interest. We address ourselves, not to their humanity but to their self-love, and never talk to them of our own necessities but of their advantages. Übersetzung aus: Nutzinger, 1991, S.80, Fußnote 3, WN, I.ii.2,17. 
Konsumgüter, vor allem für Ess/Trinkbares, Nahrungs-, Genuss- und Heilmittel sich einer moralisch-religiösen Regelung unterzieht oder nicht, ob moralisch-religiöse Gebote und Verbote geachtet oder übertreten werden. In diesem Bezug, intressierte mich das Verhältnis zwischen Motiviertheit der Nomination und Marketing, ohne von speziellen Interessen im Bereich von Religion und Finanzen bewogen zu sein, oder besonders weil ich weder eine religiöse noch eine finanzielle Bildung genossen habe.

Für Plato ist die Kenntnis von Natur, Umwelt und spezifischen Merkmalen der Dinge für ihre Benennung von Bedeutung ersten Ranges; sein Kratylos weist nur zu gut nach, wie alt das philosophisch-linguistische Thema der Namensgebung ist, wie motiviert / unmotiviert Benennungen sind, und im Allgemeinen, wie wichtig es ist, Umwelt - Natur und Gesellschaft - zu kennen, um Dinge zu benennen. Außerdem, finden fast alle Fragen, die sich Sprachwissenschaftler im Laufe der Zeit von der Antike bis zur Gegenwart, gestellt haben, im Kratylos $\left(\right.$ Kratylos) ${ }^{2}$ von Plato eine Antwort.

\section{Gesellschaft als Kommunikationssystem und binäre Codierung der Sprache, Religion und Moral nach Luhmann}

Basierend auf der Systemtheorie, erfasst der Soziologe Niklas Luhmann ${ }^{3}$ die Gesellschaft als Kommunikationssystem, als ein Funktionssystem, in dem Sprache binär codiert ist, was bedeutet, dass es keine anderen Möglichkeiten gibt, als Annahme oder Ablehnung bzw. Ja/Nein-Situationen. "Jedes ausgesprochene Wort erregt den Gegensinn" schrieb auch Goethe in den Wahlverwandschaften. "Will man dieses Risiko vermeiden, muß man auf Kommunikation verzichten." - ist Luhmanns Meinung 5 . Die theoretischen Grundlagen der obigen Hypothese liegen in der Annahme „daß die Gesellschaft ein auf der Basis von Kommunikation operativ geschlossenes Sozialsystem ist und daß deshalb ihre Evolution den Problemen der Autopoiesis von Kommunikation folgt, die ihrerseits in ihren Bedingungen durch die Evolution

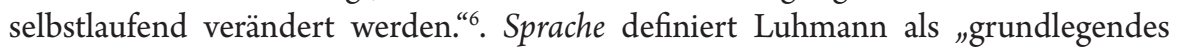
Kommunikationsmedium, das die reguläre, mit Fortsetzung rechnende Autopoiesis der Gesellschaft garantiert"?

2 Platon: Sämtliche Werke. Band 1, Berlin [1940], S. 541-617.Entstanden zwischen 393 und 388 v. Chr. Erstdruck (in lateinischer Übersetzung durch Marsilio Ficino) in: Opera, Florenz o. J. (ca. 1482/84). Erstdruck des griechischen Originals in: Hapanta ta tu Platônos, herausgegeben von M. Musoros, Venedig 1513. Erste deutsche Übersetzung durch J. G. Zierlein in: Magazin der deutschen Kritik, herausgegeben von C. von Schirach, Band 1/2, Halle 1772. Der Text folgt der Übersetzung von Julius Deuschle von 1855. Permalink: http://www.zeno. org/nid/20009262520 Lizenz:Gemeinfrei Kategorien:Attische Philosophie.

3 Luhmann, Niklas: Die Gesellschaft der Gesellschaft, Frankfurt a. M.: Suhrkamp 1997.

4 aus Ottiliens Tagebuch, in: Die Wahlverwandtschaften, zit. nach: Goethes Werke (Hrsg. Ludwig Geiger) 6. Aufl. Berlin1893, Bd. 5, S. 500 Fussnote 314 in Luhmann, 1997, S.102

Luhmann, 1997, S.102.

6 Luhmann, 1997, S.93.

7 Luhmann, 1997, S.339. 
Die Autopoiesis besteht in der Reproduktion (=Produktion aus Produkten) der elementaren Operationen des Systems, also zum Beispiel von Zahlungen, von Rechtsbehauptungen, von Kommunikation über Lernleistungen, von kollektiv bindenden Entscheidungen usw. Die distinkte Qualität solcher Elementaroperationen, ihre Unverwechselbarkeit im Verhältnis zu den Elementen anderer Systeme, liegt darin begründet, daß sie im Kontingenzbereich eines spezifischen Codes konstituiert sind (und nicht etwa darin, daß sie dessen positiven Wert bezeichnen). Sie sind stets formbezogen produziert. Auch Unrecht ist durch das Rechtssystem, auch Unwahrheit ist durch das Wissenschaftssystem determiniert, und der Code schließt nur dritte Möglichkeiten aus. Durch alle Operationen des Systems wird der binäre Code (mitsamt dem Ausschluß dritter Werte) laufend reproduziert, und mit den dadurch immer neu möglichen eigenen Operationen erfüllt das System seine Funktion (und nicht etwa darin, daß sie dessen positiven Wert bezeichnen). Codes sind aber nicht Abbilder einer Wertwirklichkeit, sondern einfache Duplikationsregeln. Sie stellen für alles, was in ihrem Anwendungsbereich (den sie selbst definieren) als Information (die sie selbstkonstituieren) vorkommt, ein Negativkorrelat zur Verfügung. Also etwa: wahr/unwahr; geliebt/nicht geliebt; Eigentum haben/nicht haben; Prüfungen bestehen/nicht bestehen; Amtsmacht ausüben/ihr unterworfen sein usw. Daraufhin erscheint alles, was mit der Form des Codes erfaßt wird, als contingent - als auch anders möglich. In der Praxis entsteht damit ein Bedarf für Entscheidungsregeln, die festlegen, unter welchen Bedingungen der Wert bzw. der Gegenwert richtig bzw. falsch zugeordnet ist. Wir nennen solche Regeln Programme. Die Unterscheidung von Codes und Programmen strukturiert [...] die Autopoiesis der Funktionssysteme in einer unverwechselbaren Weise, und die daraus resultierende Semantik unterscheidet sich grundlegend von den Teleologien, Perfektionsvorstellungen, Idealen oder Wertbeziehungen der Tradition. ${ }^{8}$

Religion und Moral sind Subsysteme, die auf Grund von Konditionierungen und Regelungen der Gesellschaft entstehen, wie auch Geheimhaltung, Tabus, Sakralisierung in der Zeit vor der Schaffung der Schrift bis zur Überlappung von Religion und Moral bzw. das, was richtig oder falsch im Verhalten des Menschen ist.

Vor allem geht es um einen im Verhältnis zur Sprache neuartigen Code, nämlich um die Unterscheidung von gutem und schlechtem Verhalten. Wie der Sprachcode selbst enthält auch dieser Code nur zwei Werte, und ebenfalls einen positiven und einen negativen Wert. Der Moralcode steht aber quer zum Sprachcode mit der Folge, daß sowohl das Annehmen als auch das Ablehnen einer Kommunikation sowohl gut als auch schlecht sein kann. Darin liegt, verglichen mit der [...] Restriktion der Kommunikation, die Unwahrscheinlichkeit der Moral und speziell die Unwahrscheinlichkeit, daß die durch die Sprache freigesetzten Risiken auf diese Weise kontrolliert werden können. ${ }^{9}$

Zum Vergleich mit der Tradition: im Talmud wird vor dem möglichen negativen Wert von ausgedrückten Gedanken, Handlungen, Gewohnheiten und Charakter, das alles zur Bildung des menschlichen Schicksals führt, vorgewarnt:

8 Luhmann, 1997, S. 339-341

9 Luhmann, 1997, S.110. 
Achte auf deine Gedanken, denn sie werden Worte.

Achte auf deine Worte, denn sie werden Handlungen.

Achte auf deine Handlungen, denn sie werden Gewohnheiten.

Achte auf deine Gewohnheiten, denn sie werden dein Charakter.

Achte auf deinen Charakter, denn er wird dein Schicksal. ${ }^{10}$

Unwahrscheinlich ist es aber, dass die sprachliche Restriktion die Risiken kontrollieren kann. Demnach, soll dasselbe auch für den Gebrauch von Wendungen wie, beispielsweise „Gottseibeiuns " ${ }^{\text {"11 }}$ oder rum. „uciga-l toaca“12 ${ }^{\text {"12 }}$ gelten.

Nach der „Einführung des Buchdrucks lockert sich auch der Zusammenhang von Religion und Moral“, meint Luhmann, und bietet das Beispiel der religiösen Bürgerkriege, „die auf beiden Seiten mit moralischem Eifer ausgefochten werden“, nach denen im 17. Jahrhundert „die psychologische, im 18. Jahrhundert die begründungstheoretische Problematisierung der Moral“ folgt. Die Religion wird „nicht mehr als Einteilung der Welt vorgestellt, die es kommunikativ auf angemessene Weise nachzuvollziehen gilt, sondern als Kommunikation besonderer Art mit besonderen Sinngehalten und besonderen Funktionen“ und sie „erscheint jetzt als eine reduktive Struktur besonderer Art, also als kontingent“. Der Mensch „,ist nicht durch sie [Religion] gebunden, weil man anderenfalls in Irrtum und Sünde leben würde. Man kann an sie glauben - oder auch nicht ${ }^{13}$ Für Luhmann befindet sich die Moral in der heutigen Gesellschaft in einer prekären Lage. Ihr

entspricht, auf semantischer Ebene, die Individualisierung der moralischen Referenz, ihr Insistieren auf innerem Überzeugtsein (im Unterschied zu äußerem Gezwungensein),

10 https://www.aphorismen.de/zitat/19331

11 Prophylaktisch gebrauchter Ausruf, zur Abwehr schlimmer Folgen, wenn man vom Teufel sprach u. um den göttlichen Beistand zu erbitten; n.Wiktionary, siehe Quellen; weiter: Umschreibungen und verhüllende Bezeichnungen: manche glauben, dass die Nennung des Namens des Teufels dessen Beschwörung werden könne. Daher die Vielzahl verhüllender Bezeichnungen und Umschreibungen für den Teufel. Ein anderer Grund zur Umschreibung: die Betonung mancher charakteristischen Merkmale. Beispiele: Leibhaftiger, Gottseibeiuns (volkstümlich); Daus (volkstümlich, veraltet), enthalten in der Wendung „ei der Daus“ Widersacher; Verführer; Höllenfürst; Kuckuck („Hol’s der Kuckuck“); Höllenwart ([...] alteTeufelsbezeichnungen „Hellewart“, „Hellewirt“, „Hellehirt“); Fürst dieser Welt; Sohn der Verdammnis; gefallener Morgenstern; Herr der Fliegen (wörtl. Übersetzung von hebr. Beelzebub); Tausendkünstler (lat. milleartifex); zusammengefasst, abgek. nach: https://de.wikipedia.org/wiki/Teufel

12 Ucigă-l-toaca Sinonime: aghiuță, alcor, demon, diavol, drac, încornoratul, naiba, necuratul, satană, tartor. Drac Sinonime: diavol, demon, necuratul; rău, nestăpânit, crud; aghiuță, încornoratul, naiba, satană, tartor, belzebut, scaraoțchi, michiduță, nichipercea, pârlea, sarsailă, faraon, idol, împelițatul, mititelul, nefârtatul, nevoia, pârdalnicul, procletul, pustiul, vicleanul, cel-de-pe-comoară, cel-din-baltă, ducă-se-pe pustii, ucigă-l-crucea, ucigă-toaca, mamon, săcretul, sotea, hâdache, năpustul, spurc, spurcat, șeitan, șotcă, ucigan, bedă, benga, carcandilă, mutul, pocnetul, sarsan, scaloi, șlactrafu, împiedicătorul, nepriitorul, păcatul; poznaș, isteț, vioi, al naibii; http://dex.dictoo.eu/index.php?cheie=(ucig\%C4\%83-1-toaca)\&

13 Luhmann, 1997,S.113. 
also auf Selbstmotivation. Diese Individualethik wird von Religion abgekoppelt und von Recht unterschieden. Das läßt die Frage offen, wie es dann überhaupt zu einer sozialen Koordination moralischer Perspektiven kommen kann. Wenn heute überall - in der Wirtschaft, in der Politik, in ökologischen Fragen für Ärzte, für Journalisten - nach „Ethik“ verlangt wird, vermißt man die Durchpräzisierung der Frage im Hinblick auf die sozialen Mechanismen, die eine solche dann unmoralische Koordination der Moral bewirken könnten. ${ }^{14}$

\section{Gedanken über Sakralität, Glaube, Tradition und soziale Handlung}

Die Welt des Glaubens (der Religion), gleich ähnlich wie die der Wirtschaft wendet sich an ihre Zielgruppen, möchte ihre Produkte gewinnbringend absetzen, die Kunden für sich gewinnen und an sich binden. Dies wird mit Hilfe raffiniert gearteter Strategien und Taktiken durchgeführt, wie auch mit Hilfe vielfältiger materieller Mittel wie Ikonen, Kruzifixe, Rosenkränze, Anhänger bis T-shirts mit biblischen Sprüchen, Fläschchen mit Weihwasser, Souvenirs von Pilgerorten u.ä.

The study of religion and materiality is an important and fast-growing sub-discipline in the contemporary Religious Studies scene. According to the editors of the premier journal in this area, the aptly named 'Material Religion', scholars in this area explore how religion happens in material culture - images, devotional and liturgical objects, architecture and sacred space, works of arts and mass-produced artifacts. No less important than these material forms are the many different practices that put them to work. Ritual, communication, ceremony, instruction, meditation, propaganda, pilgrimage, display, magic, liturgy and interpretation constitute many of the practices whereby religious material culture constructs the worlds of belief. ${ }^{15}$

Umgekehrt, bietet die Wirtschaft, mittels Marketing und Werbung, Produkte an, welche durch ihren kulturellen und religiösen Inhalt und darauf andeutenden Namen, ebenfalls Kunden zu gewinnen und an sich zu binden versuchen. So gibt es auf dem Markt sowohl gezielt religiös-gebundene Produkte (z.B. koshere Lebensmittel, Pessach Brot, Fasten-Produkte), aus religiösen Bräuchen einst sakrale oder magische, allmählich zur Tradition gewordene Produkte (Krampus und Nikolaus, Zwetschgenkrampus in Österreich, Bayern, u.a.; in Rumänien: pupezi $^{16}, \operatorname{colac}^{17}$, colivă ${ }^{18}$, mucenici ${ }^{19}$ ) wie auch, durch die Namengebung, mit Religion / religiösen Einrichtungen und Gestalten in

\footnotetext{
14 Luhmann, 1997,S.113.

15 The Religions Studies Project, 2010, Material Religion http://www.religiousstudiesproject.com/podcast/podcast-david-morgan-on-material-religion/

16 Eine Art Krampus; das Vorbild ist der Wiedehopf.

17 Österr. Kolatsche.

18 „Totenkuchen“: ein rituales Dessert bei den orthodoxen Begräbnissen, aus gekochten Gerste /Korn, Zucker, Nüssen.

19 Kleine, etwa brezelförmige Kolatsche aus gebackenem /gekochtem Teig, mit Zucker/ Honig, Nuss und Zimt, in Verbindung mit dem 9.März, „Gedenktag für die 40 Märtyrer / Heiligen von Sebastia“ (orthodoxer Feiertag in Rumänien und der Republik Moldau)
} 

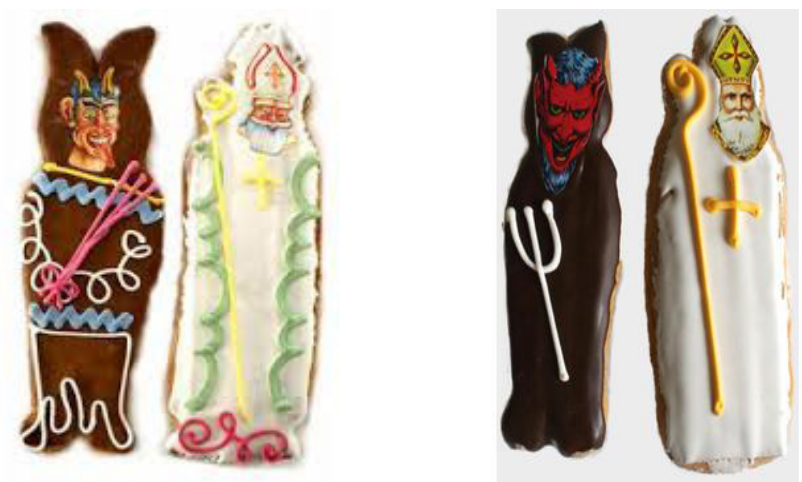

Lebkuchen Krampus und Nikolaus ${ }^{20}$

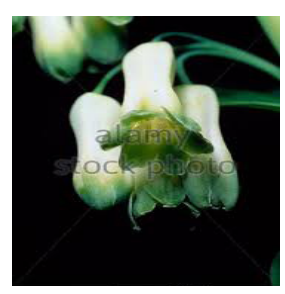

Salomos Siegel ${ }^{21}$

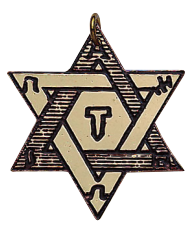

Salomos Siegel ${ }^{22}$

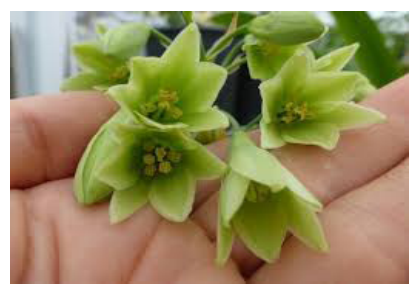

Salomos Siegel ${ }^{23}$
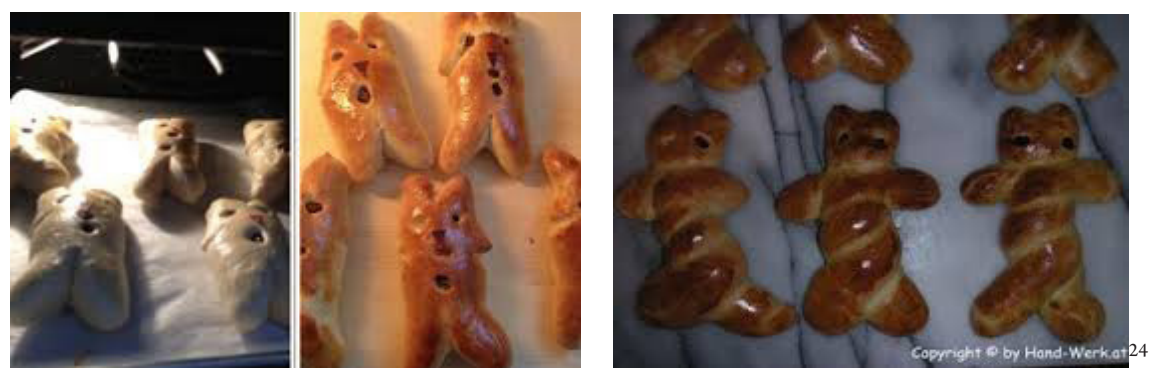

Krampuskuchen ${ }^{25}$

20 https://www.shoepping.at/c/kuchen-co

21 Eckige Salomonssiegel (Polygonatum Odoratum oder Polygonatum Officinale), Liliaceae. Fotograf: Universal Images Group North America LLC / DeAgostini / Alamy Stock Foto https: / /www.google.ro/search?q=salomos+siegel\&rlz=1C1AOHY_roRO708RO708\&tbm=i $\mathrm{sch} \& \mathrm{tbo}=\mathrm{u} \&$ source $=$ univ\&sa $=$ X\&ved $=0 \mathrm{ahUKEwie} 7 \mathrm{uDnitLXAhVH} 46 \mathrm{QKHRV} 1 \mathrm{DyEQsAQI}$ UQ\&biw $=1440 \&$ bih $=805 \#$ imgrc $=$ M5dNIzhSURsW_M:

22 http://www.amalet.de/Salomos-Siegel-2

$23 \mathrm{http}$ : / / www.hausgarten.net/gartenforum/attachments / pflanzenallgemein/412906d1408004992-gruene-blueten-p1180398.jpg

${ }_{24}$ http://www.freudeamkochen.at/category/rezepte/kuchen-torten/page/6/

25 https://www.google.ro/imgres?imgurl=https\%3A\%2F\%2Fwww.haubis.com\%2Fsi 
Verbindung gebrachte Natur- oder Kultur-Produkte (z.B. dt.: Götterspeise; Abteibier, Klosterfrau Melissengeist; rum. vin/pui mânăstiresc [Klosterwein, -hähnchen]; frz. pets de nonne, dt. Nonnenfürzle, Trappist) bis zu anderen, viel komplexer gestalteten Nominationen, welche, aus der Sicht der Religion, Positives und Negatives vereinen z.B. Himmel und Hölle.)

„Im Sakralen liegt daher zunächst keine Garantie für Dauer, für Verehrung, für Tradition, und wenn es zur Tradition wird, ist dies schon der erste Schritt zur Auflösung seiner Sakralität. ${ }^{\text {“6 }}$ - schreibt Luhmann.

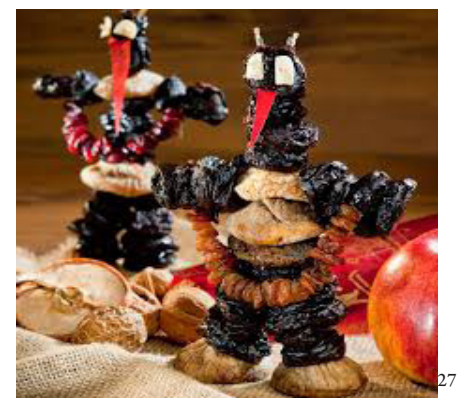

Pflaumentoffel (Teufel) (Deutschland, Österreich

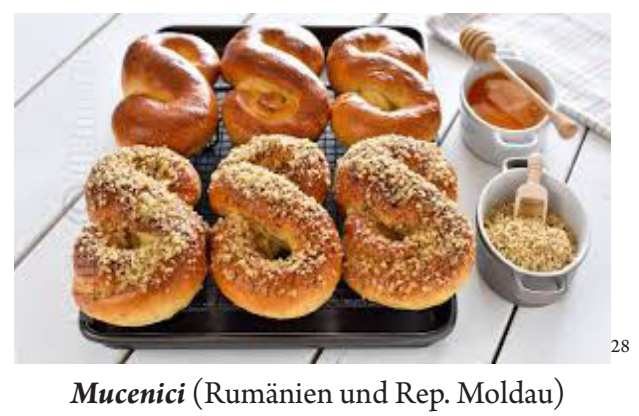

tes\%2Fdefault\%2Ffiles\%2Fstyles\%2Farticle-image-big\%2Fpublic\%2Fbrioche_krampus6. jpg\&imgrefurl=https\%3A\%2F\%2Fwww.haubis.com\%2Fit\%2Fnode\%2F961\&docid=cAzyZftz 1 xivAM\&tbnid=cILQNl7iup5FyM\%3A\&vet=10ahUKEwiW1KrVls3XAhVlApoKHUf2ANc 4ZBAzCD4oOzA7..i\&w=651\&h=407\&itg $=1 \& b i h=805 \& b i w=1440 \& q=K r a m p u s k u c h e n \& v e$ $\mathrm{d}=0$ ahUKEwiW1 KrVls3XAhVlApoKHUf2ANc4ZBAzCD4oOzA7\&iact $=$ mrc\&uact $=8$

26 Luhmann, Niklas: Die Gesellschaft der Gesellschaft, Frankfurt a. M.: Suhrkamp 1997, S.115; s.a.http://differenzen.univie.ac.at/bibliografie_literatursuche.php?sp=527

27 https://www.google.ro/search? rlz=1C1AOHY_roRO708RO708\&biw $=1440 \&$ $\mathrm{bih}=805 \& \mathrm{tbm}=\mathrm{isch} \& \mathrm{sa}=1 \& \mathrm{ei}=-\mathrm{dISWp} 7 \mathrm{fOKn}-6 \mathrm{ASkuLf} 4 \mathrm{AQ} \& \mathrm{q}=$ Krampuskuchen \&oq $=$ Krampuskuchen\&gs_l=psyab.12..0i13i30k1.23564.25051.0.26746.6.6.0.0.0.0.164.860 $.0 j 6.6 .0 \ldots . . .0 .1 .1 .64$. psy-ab..0.6.860...0i13k1.0.11XbImHmOqc\#imgdii=XfQcZGrceyxRYM:\&i mgrc $=\mathrm{xGJwUW}$ AMFPqoM:

28 https://www.google.ro/search?q=mucenici\&rlz=1C1AOHY_roRO708RO708\&tb $\mathrm{m}=\mathrm{isch} \&$ source $=\mathrm{iu} \& \mathrm{pf}=\mathrm{m} \& \mathrm{ict} \mathrm{x}=1 \&$ fir $=\mathrm{SXos} 4 \mathrm{qU} 0 \mathrm{mXVY} 2 \mathrm{M} \% 253 \mathrm{~A} \% 252 \mathrm{Cgp} 5 \mathrm{aRDpSotz}$ 

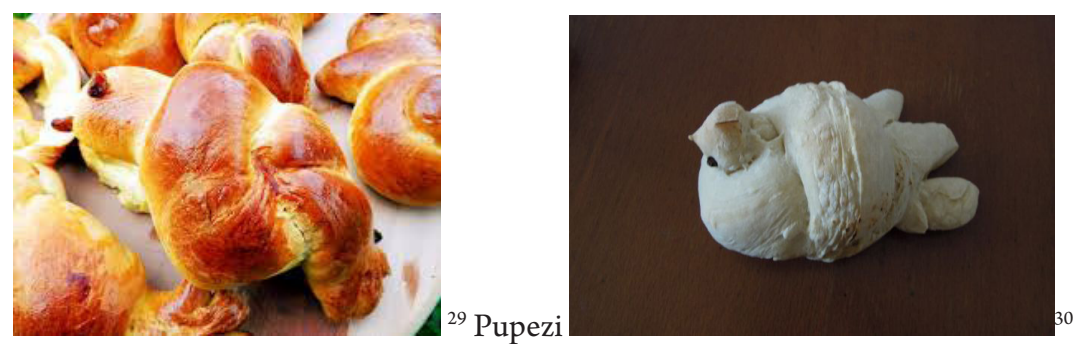

„Menschliche Kommunikation ist eine soziale Handlung der Vermittlung individueller Bedeutungskonstruktionen" schreibt Jörg Tropp ${ }^{31}$.

Inhärent jeder Kommunikation als soziale Handlung ist die Intention des Ereichens des allgemeinen Kommunikationszieles der Verständigung und bezieht sich auf Intentionen der Akzeptanz, Zustimmung oder des Für-wahr-Habens dessen, was der Kommunikationspartner mitteilt; und dieser verfolgt bestimmte Kommunikationszwecke. $^{32}$

Tropp unterscheidet 4 Typen des sozialen Handelns: das traditionale Handeln: aus eingelebter Gewohnheit, mit dem Charakter eines unreflektierten reagierens auf gewohnte Erlebnisse und das ähnliche affektuelle Handeln als hemmungsloses Reagieren auf ein nicht alltägliches Erlebnis oder Entladung von Gefühlslagen; und weiter, zwei rationale Typen des wert- und zweckrationales Handelns: das wertrationale Handeln - aus Überzeugung, ohne Berücksichtigung absehbarer Konsequenzen, wobei die Handlung vom Sinn ihres Eigenwertes bestimmt sei, der sich aus einer ideologischen Überzeugung ergebe und beispielsweise ethischer, ästhetischer, religiöser Art sein könne. Solches Handeln sei nicht einem Erfolg verpflichtet. Das Individuum, das zweckrational handelt, wäge die Mittel, Zwecke und Folgen seiner Handlung hinsichtlich der Vor- und Nachteile gegeneinander ab, mit dem Ziel, seine Handlung mit einem größtmöglichen Erfolg versprechenden Nutzen zu versehen. ${ }^{33}$ Zur Konstituierung der Kommunikation uM\%252C_\&usg $=$ _OHD9Kw708erwoC2DB3y7qvEm16U\%3D\&sa $=X \& v e d=0 a h U K E w-$ jPk8uFpM3XAhXCbZoKHdXYAekQ holiwEwCw\#imgrc=

29 Pupezi werden in Verbindung mit religiösen Feiertagen und mit dem Brot als Gabe Gottes auch heute noch gebacken. https://www.google.ro/search?q=pupezi+din+aluat+de+p aine\&rlz $=1 \mathrm{C} 1 \mathrm{AOHY}$ roRO708RO708\& $\mathrm{tbm}=\mathrm{isch} \& \mathrm{tbo}=\mathrm{u} \&$ source $=$ univ\&sa $=\mathrm{X} \& \mathrm{ved}=0 \mathrm{ahU}$ KEwi6ocTYqc3XAhVkEJoKHbr3ADgQsAQIJA\&biw=1440\&bih=805\#imgrc $=$ Kwb3ePYrIU OKGM:

${ }^{30} \mathrm{http}: / /$ adevarul.ro/locale/zalau/povestea-nestiuta-painii-singurul-alimentrugaciunile-crestine-mituri-ciudate-despre-darul-dumnezeu-nu-arunca-niciodata1_5543575ecfbe $376 \mathrm{e} 35 \mathrm{bcd} 11 \mathrm{~d} /$ index.html

31 Tropp, Jörg. 2014. Moderne Marketing-Kommunikation: System - Prozess - Management. Springer-Verlag.

32 Tropp, 2014, S. 28

33 Tropp, 2014. S.30 
als ein Prozess der Bedeutungsvermittlung kognitiv autonomer, handelnder Menschen, seien 3 komplementäre Kriterien notwendig, die als kommunikative Prämissen des Marketings aufgefasst werden können: Selektivität, Reflexivität, Kontextualität. ${ }^{34}$ Erst durch Selektivität werde die Komplexität der Umwelt für das Individuum behandelbar (egal ob rezipierend oder produzierend). Die Aufmerksamkeit ist selektiv und wird vor allem vom Interesse gesteuert. ${ }^{35}$ Die Reflexivität ist die unter bestimmten Bedingungen auftretende Reflektierung von Prozessen auf sich selbst, womit eine Steigerung von Leistungen einhergeht (Tropp, Definition von Klaus Merten, 1977, S.86 ${ }^{36}$ ). Reflxivität hat drei Merkmale: zeitlich (Menschen können mit altem Sprachmaterial neue Wörter und Worte bilden), sachlich (Aussagen werden immer von Meta-Aussagen begleitet, die Kommunikationsangebote erst verständlich machen) und sozial (es besteht eine wechselseitige Kopplung der Kommunikationspartner). Kontextualität der Kommunikation ist der notwendige Einfluss des Zusammenhangs, aus dem eine kommunikative Handlung einen erklärbaren Sinn bezieht. ${ }^{37}$ Wie Adam Smith schon im 18. Jh. erkannt hat, dass „Nicht vom Wohlwollen des Metzgers, Brauers und Bäckers erwarten wir das was wir zum Essen brauchen, sondern davon, daß sie ihre eigenen Interessen wahrnehmen. Wir wenden uns nicht an ihre Menschen-, sondern an ihre Eigenliebe, und wir erwähnen nicht die eigenen Bedürfnisse, sondern sprechen von ihrem Vorteil“" (S. Fußnote 1). In der Marketingkommunikation wird nicht allein Verständigung intendiert, sondern es werden „Kommunikationszwecke verfolgt, die den Grund für die Initiierung des Kommunikationsprozesses ausmachen, wie etwa die Steigerung des Bekanntgrades von Produkten, die Erhöhung der Werbeerinnerung, der Aufbau eines unverwechselbaren Markenimages oder der Kauf des beworbenen Produktes. ${ }^{\text {"38 }}$

\section{Essen und Trinken als sozio-kulturelle System und Handeln}

Der Mensch isst nicht nur um zu überleben. Seine Ernährung steht in engem Verhältnis mit allen Seiten seiner individuellen und sozialen Existenz, welche die kulturellen und ökonomischen Aspekte der Ernährung umfasst: von der wilden Stillung des Heißhungers zur Bändigung des Appetits bei zivilisierten Tischmanieren, über die soziale Funktion der Mahlzeiten, die Kulturbedingtheit der Nahrungstabus und der kulinarischen Moral, von den vielfältigen Ursachen von Hungersnot, Mangel- und Unterernährung, bis zur Ernährung im Überfluss oder als Verführung und zu Festmahl, von Fast Food und Fusion Food und Internationalität der Gastronomie bis zur rentablen Pflege der regionalen Kulinarik, der alltäglichen und rituellen Kost.

Das komplexe Thema Essen - Trinken - Kulinarik ist ein großzügiges Forschungsgegenstand für Wissenschaftler vielfältiger Bereiche, so für Nutritionisten /

\footnotetext{
34 Tropp, 2014. S.30

35 Tropp, 2014, S.34

36 Merten, Klaus. 1977. Kommunikation. Eine Begiffs- und Prozeßanalyse. Opladen.

37 Tropp, 2014, S.43.

38 Tropp, 2014, S. 28.
} 
Ernährungswissenschaftler und -psychologen, Nahrungsethnologen, Historiker und Kunsthistoriker, Anthropologen und Soziologen, Theologen und Kulturwissenschaftler. Für Sprachwissenschaftler, Psycho- und Soziolinguisten wie auch für Wirtschafts/ Marketingwissenschaftler und -praktiker spielen die kulturellen und interkulturellen Aspekte des Essens / der Ernährung und der Kochkunst und ihre interdisziplinäre Thematik eine immer wichtigere Rolle.

Essen und Trinken werden als sozio-kulturelles System und Handeln betrachtet und diachronisch und synchronisch studiert. Erst im 20. Jh. wurde die ausschließlich naturwissenschaftliche Sicht überwunden ${ }^{39}$. In Günter Wiegelmanns (1928-2008) „Alltagsund Festspeisen. Wandel und gegenwärtige Stellung" (1967) werden wirtschaftliche, lebensmitteltechnische und medizinische Aspekte auch aus dem historisch-soziokulturellen Blickwinkel analysiert, wobei soziale Verzehrssituation und gemeinsame Mahlzeit im Zentrum des Forschungsinteresses stehen. Ulrich Tolksdorf (1938-1992) spricht über Mahlzeit als Speise und soziale Situation: welches Lebensmittel unter welchen gesellschaftlichen Umständen wird verzehrt. Günter Wiegelmann und Hans Jürgen Teuteberg führten den Begriff Nahrungsforschung ein. Für den Strukturalisten Claude Lévi-Strauss (1908-2009) ist die Esskultur Veranschaulichung der Struktur einer Gesellschaft, welche das bestimmt, was Lebensmittel sind, was essbar ist / was / wann gegessen wird. Damit wird aus der Sicht der Kommunikationswissenschaft der Laswell-Formel genuggetan: Wer sagt was in welchem Kanal zu wem mit welchem Effekt? (Who says what in which channel to whom with what effect? $)^{40}$ : Was für ein Lebensmittel wird wann, in welcher Situation / welchen sozialen Umständen, zu welchem Zweck, mit welchem Effekt verzehrt? Die Auswahl der Lebensmittel ist u.a. abhängig von: ökologischen-regionalen Bedingungen, sozialer Normierung, Weltanschauung und Glaubensbekenntnis. „Die Mahlzeit erfüllt alle Bedingungen, die man an eine Grundeinheit stellen kann: Sie ist zu allen Zeiten und in allen Sozialgruppen gleichermaßen vorhanden. ${ }^{\text {"41 }}$ Dauer, Inhalt, Zubereitung,Tageszeit der Mahlzeit wandelten sich im Laufe der Jahrhunderte.

39 Nahrungsforschung, in Kunst- und Kulturwissenschaft online (C) Sandy Alami 2015; angegebene Literaturauswahl: Abel, Wilhelm: Stufen der Ernährung. Eine historische Skizze, Göttingen 1981;Teuteberg, Hans Jürgen/ Wiegelmann, Günter: Unsere tägliche Kost. Geschichte u. regionale Prägung, Münster 1986;Tolksdorf, Ulrich: Nahrungsforschung. In: Brednich, Rolf [Hrsg.]: Grundriss der Volkskunde. Einführung in die Forschungsfelder der europäischen Ethnologie, Berlin 1988;Wiegelmann, Günter: Volkskundliche Studien zum Wandel der Speisen und Mahlzeiten. In: Teuteberg, H.J./Wiegelmann, G.: Der Wandel der Nahrungsgewohnheiten unter dem Einfluß der Industrialisierung, Göttingen 1972; Wiegelmann, Günter; Krug-Richter, Barbara [Mitarb.]: Alltags- und Festspeisen in Mitteleuropa. Innovationen, Strukturen und Regionen vom späten Mittelalter bis zum 20.Jahrhundert, Münster [u.a.] 2006. Kunst- und Kulturwissenschaft online www.kukwiso.com/index.php/ de/nahrungsforschung//

40 Beck, Klaus. 2013. Lasswell-Formel. In Lexikon Kommunikations-und Medienwissenschaft. 2. Auflage, Günter Bentele, Hans-Bernd Brosius, Otfried Jarren (Hrsg.), 182. Wiesbaden: Springer Fachmedien.

$41 \quad$ Teuteberg/Wiegelmann 1986, S. 29. 
Mit Wilhelm Abels (1904-1985) Stufen der Ernährung (1981) ${ }^{42}$ wurden die zwei Stufen der Ernährung von Adam Smith (1723-1790) - Fleisch und Getreide - um eine dritte erweitert, die der Veredelungswirtschaft von heute entspricht. ${ }^{43}$

\section{Zum Interesse des Namensgebers zwischen Natur und Kultur}

Veredelungswirtschaft bedeutet:

Produktveredelung, bewirkt durch eine substanziell meist unerhebliche technische Veränderung, Form- und (oder) Qualitätsverbesserungen, die nicht zu einer eigentlichen Stoffumwandlung führen, die aber für eine zweckmäßigere Weiterverarbeitung oder, bei Fertigerzeugnissen, für einen individuell verfeinerten Geschmack wirtschaftlich bedeutungsvoll sind. ${ }^{44}$

Anscheinend sind Birnen, zum Beispiel, Naturprodukte im Gegensatz zu Kulturprodukten bzw. von Menschenhand und -geist geschaffenen materiellen und imateriellen Gütern. Nach langzeitiger Verbesserung einer oder der anderen Eigenschaft der Birne durch menschlichen Eingriff, würde ich sie gerne als NaturKultur-Produkt ansehen. In Natur als Kulturprodukt Kulturökologie und Umweltethik von David Krieger und Christian Jäggi ${ }^{45}$ werden die Forschungsergebnisse eines Projektes mit dem Schwerpunkt Umwelt veröffentlicht, welche den Zusammenhang zwischen Natur und Kultur erkennbar werden lassen. Da die Umweltforschung lange schon nicht mehr „ausschließliche Fragestellung der Umweltforschung oder gar Eigentum der Naturwissenschaften“ sein soll, „werden Natur und Kultur als soziales System aufgefasst. Umweltfragen, ihre Thematisierung oder Ignorierung sind Ausdruck gesellschaftlicher Kommunikation, bzw. der Art, wie eine Gesellschaft organisiert ist" ${ }^{46}$

Am Beispiel von Benennungen der über 2900 offiziell registrierten Birnensorten die ich nun aus der Sicht der Natur und Kultur als soziales System anschaue, bringe ich weiter unten einige Beispiele (in Verbindung mit dem Thema heilig und weltlich) zum engen Verhältnis zwischen Nomination von Lebensmitteln, soziokultureller Entwicklung und gesellschaftlichem Interesse für Namen / Namensgebung wie auch zu Prinzipien der Nomination, denen Merkmale der durchquerten historischen Epochen abzulesen sind.

\begin{tabular}{|c|c|}
\hline 1. Gott, Christus & Williams Christ Birnen / rum. pere vilmoșe; Ah-mon-Dieu \\
\hline 2. Engel & Angelys $^{1}$ \\
\hline 3. Anonyme Gläubige & Bon Chrétien d'Hiver (schon bei den Römern, dann XVII.Jh.) \\
\hline 4. Religiöse Feiertage & Belle de Noel², Fondante de Noel, Beurrée d’Assomption \\
\hline
\end{tabular}

${ }^{42}$ Abel, Wilhelm, Stufen der Ernährung. Eine historische Skizze, Verlag Vandenhoeck 1981.

43 www.kukwiso.com/index.php/de/nahrungsforschung

44 Gabler Wirtschaftslexikon http://wirtschaftslexikon.gabler.de/Definition/veredelung.html\#definition

45 Erschienen 1997 bei Birkhäuser Basel.

46 http://www.springer.com/de/book/9783764354886\#aboutBook 


\begin{tabular}{|c|c|}
\hline 5. Heilige & $\begin{array}{l}\text { Santa Maria (GB); Sainte Anne; Sainte Dorothée; Santa } \\
\text { Claus; Saint- Rémy; Saint- Michel Archange; Saint-Mathieu / } \\
\text { Joseph / Ghislain / Germain / André }\end{array}$ \\
\hline 6. Kirchenränge & $\begin{array}{l}\text { Abbé Adrien Grégoire }{ }^{3} \text { / Abbé / Abate Fétel (FR 1866) }{ }^{4} \text {; } \\
\text { Cardinal Georges d'Amboises ; Soeur Grégoire (Belgium } 1858 \\
\text { M. Grégoire Nélis) }{ }^{6} \text {; Bishop's Thumb; Monseigneur Affre }\end{array}$ \\
\hline $\begin{array}{l}\text { 7. Weltliche Persönlichke- } \\
\text { iten, } \\
\text { Adelstitel, Ränge, Berufe, } \\
\text { Stellungen }\end{array}$ & $\begin{array}{l}\text {-Henri IV, Pierre Corneille, Napoléon, Jeanne d’Arc, Ferdi- } \\
\text { nand de Lesseps, Richelieu •Reine des Belges, Prince, Prinzes- } \\
\text { sin Marianne, duchesse, comtesse, marquise, seigneur Esperen, } \\
\text { madame, Königin, Herzogin, duc •ministre docteur, general, } \\
\text { directeur, curé, avocat, professeur, président, conseiller a la } \\
\text { cour, commissaire, urbaniste, doyenne, senateur notaire Lepin, } \\
\text { Soldat laboureur }\end{array}$ \\
\hline 8. Toponyme & $\begin{array}{l}\text { Someşana (RO), Napoca (RO), Europa, Corse, Columbia, } \\
\text { Cluj (RO), Bristol, Cross, Aramiu de Someş (RO), Hollande, } \\
\text { Bruxelles, Anjou, Angleterre, Babylone, Angevine, Orient, } \\
\text { Oregon, Roma }\end{array}$ \\
\hline 9. Ereignisse & Congrès, Conférence \\
\hline $\begin{array}{l}\text { 10. Körperliche/Soziale } \\
\text { Besonderheiten/Nachteile }\end{array}$ & nain, orpheline \\
\hline 11. Staat/Politik & Republica, royale \\
\hline 12. Reliefformen/Natur & Cascade [Wasserfall] \\
\hline 13. Tiere & chat $[$ Katze] \\
\hline 14. Pflanzen & Calebasse [Klobasse, eine Art Kürbis], Courge [Kürbis] \\
\hline 15. Metalle & Bronzée, Argent \\
\hline $\begin{array}{l}\text { 16. Farben, Jahreszeiten, } \\
\text { haptische (taktile) Wahr- } \\
\text { nehmung u.a. }\end{array}$ & Bronzée, Argent, d'Hiver, Fondante, Beurée \\
\hline
\end{tabular}

http://pomologie.com/poire/poire1/fpoires/varietes.html

${ }^{1}$ (neuere Sorte) http://pomologie.com/ ${ }^{4}$ https://it.wikipedia.org/wiki/ poire/poire1/fpoires/angelys/angelys.html by Abate_F\%C3\%A9tel) http://www.pepinierINRA

${ }^{2} \mathrm{http}$ ://pomologie.com/poire/poire1/ ${ }^{5} \mathrm{http}: / /$ pomologie.com/poire/poire1/ fpoires/fondantedenoel/fondantedenoel. fpoires/cardinalgeorgesdamboise/cardinalhtml 1842

${ }^{3} \mathrm{http}: / /$ pomologie.com/poire/poire1/ ${ }^{6} \mathrm{http}: / /$ pomologie.com/poire/poire 1/ fpoires/abbefetel/abbefetel.html fpoires/soeurgregoire/soeurgregoire.html

Einige hunderte von Birnensorten tragen Namen der Sortenschöpfer oder andere Personennamen, als hier unter 5., 6. Und 7. zum Beispiel angeführt. Die meisten Namen von Birnensorten enthalten Anthroponyme (Namen kirchlicher und weltlicher, die Mehrheit, aber weltlicher Personen) und Toponyme, sprachliche Mittel durch welche der Benenner / Namensgeber die besondere Bedeutung seines sozialen Umfeldes in Verbindung mit dem Natur-Kulturprodukt unterstreicht. 


\section{Vom Essen und Trinken in der Bibel und im Marketing - Gesellschaftliches, Signifizierendes, Moralisches, Kommunikatives, Gewinnbringendes}

Die Hauptgetränke und -lebensmittel in der Bibel sind: Wasser, Milch, Wein; Salz, Honig,Trauben, Öl, Getreide, Hülsenfrüchte, Gerste, Hirse, Linsen, Bohnen, Brot, Knoblauch, Zwiebeln, Lauch, Gurkengewächse, Kapern, Feigen, Oliven, Datteln, Granatäpfel, Nüsse, Pistazien, Mandeln, Melonen, Rosinen, Kümmel, Koriander, Safran, Zimt, Essig, Butter, Käse, Fleisch, Fisch. ${ }^{47}$ Das bedeutet nicht, dass es keine „Leckereien” gibt, von denen aber eher abgeraten wird. 28 Stellen im Buch der Sprichwörter beziehen sich auf Genuß und Produktion von Lebensmitteln, fast alle in Verbindung mit sozialen Verhältnissen, Religion u./o. Moral. Ursprung und Vorhandensein von Lebensmitteln werden mit Wirtschaft und Sinn für Wirtschaft korreliert. Viele Bezüge betreffen Nahrungsaufnahme als natürliche, unverzichtbare Möglichkeit zur Aufrechterhaltung der Lebensfunktionen des menschlichen Körpers, aber auch als soziale Manifestierungen wie Gastfreundschaft, Ehr- und Schutzerbietung, geistig-kulturelle Ereignisse (Massenspeisungen, das Abendmahl zum Gedenken an Jesus, das Hochzeitsmahl als immer wieder inszenierte Bild des himmlischen Heils / der Freudenzeit des Himmels.

In den biblischen Sprüchen werden Speisen und Getränke in Verbindung mit Empfehlungen im Bereich der sozialen Beziehungen, einschließlich der interpersonalen Kommunikation, mit den zu pflegenden sozio-kulturellen und moralischenWerten aus der Sicht der christlichen Religion ebenfalls antithetisch, oppositiv dargestellt; Speisen und Getränke dienen zur doppelten Erläuterung von Abstrakta-Paaren (durch die Nennung der Begriffe und die umgekehrte Korrelation ihrer positiven vs. negativen Merkmale):

\footnotetext{
•.esser: trockener Bissen (und Ruhe) vs. Festspeisen (und Streit) (17:1); Gemüse (und Liebe) vs. gemästeter Ochse (und Hass)

- Gastfreundschaft $\rightarrow$ dem Fremden,: Honig, Öl (5:3); Brot, Wein (9:5); dem Hasser $\rightarrow$ Brot, Wasser (25:21)

-Weisheit: Schlachtvieh, Wein (9:2);

-schlecht: vornehm tun, aber kein Brot (1:9);

-Mangel durch Festfreude, nicht reich: Wein, Öl (wer liebt) (21:17)

-gut: Ackerland bebauen, Brot essen vs. nichtigen Dingen jagen (1:11)

-nicht begierig sein, nicht essen vom Missgünstigen: Leckerbissen, trügerische Speise, Brot $(23: 3 ; 23: 6)$

-nicht begierig sein, nicht satt werden, nicht ausspeisen: Honig (25:16); sparsam sein: Honig essen, ehrende Worte gebrauchen (25:27); Verhalten: der Satte tritt Honig mit Füssen, dem Hungrigen ist alles Bittere süss (27:7)

-genug ist da: Ziegenmilch (27:25), Kräuter der Berge (27:27)

-bei schlechtem psychischen Zustand: Rauschtrank (dem Mutlosen), Wein (dem Verbitterten) $(27: 6)$
}

47 Beispielstellen in der Bibel: Mt 4,4; Mt 5,13; Mt 6,11; Mt 14,17; Mt 25,35; Joh 2,1; Joh 6,35; u. v. a. Die Bibel nach Martin Luthers Übersetzung, revidiert 2017, (C) 2016 Deutsche Bibelgesellschaft, Stuttgart.https://www.bibleserver.com/text/LUT/Matth\%C3\%A4us4 
Mit der Evolution der Gesellschaft bis zu ihrer heutigen Komplexität entwickelten sich auch Lebensmittelverbrauch und -genuß von ihrem Ziel zur Aufrechterhaltung der Lebensfunktionen in Richtung Konvivialität und Beisammensein von Individuen und Gruppen, zum Anzeigen unterschiedlicher Zugehörigkeiten und Stellungen in der Gesellschaft und deren Möglichkeiten sich auszudrücken und durchzusetzen. Immer komplizierter und raffinierter wurden Gewinnung und Zubereitung von Lebensmitteln / Speisen / Gerichten und dadurch auch die Ansprüche der Konsumenten. Daher werden Anbieter, die sich in ständiger Marktkonkurrenz befinden, gezwungen, immer raffiniertere Werbung für ihre Produkte zu schaffen und anzuwenden.

Mit Sprachkommunikation im Bereich der Wirtschaft / des Handels befassen sich Marketing-Fachleute, mit der professionellen Namensgebung, Sprach- und Marketingmenschen mit Namensfindungs-Know-how ${ }^{48}$, denn ein gut ausgewählter / getroffener, passender, Interesse erweckender Name für ein bestimmtes Produkt ist marktorientiert und wirkt sich wie Werbung aus, der den Adressaten/die Zielgruppe zum Handeln anregen soll. Mit anderen Worten, der Adressat soll zum Nutznießeroder Konsumenten werden, indem er zugreift / kauft / bestellt / nachmacht. „Wenn die Sprache nicht stimmt, ist das was gesagt wird nicht das was gemeint ist“ sagte Konfuzius ${ }^{49}$ und manchmal liegt einer oder der andere ganz daneben mit der Benennung von Produkten, Läden usw., welche nicht intendierte, zufällige, zynisch-humoristische Effekte erzeugen kann, wie z.B. in Bukarest, wo man Lebensmittel von Angst [Ladenkette] kaufen kann, sich von Incomod [unbequem] (Bekleidungsfirma) anziehen, mit dem Dacia-Duster [Staub] fahren, sich vom Richter [Apotheke] behandeln lassen und bei Daimon [Sportclub] körperlich fit werden.

Aus den hunderten von Sites, welche die Marketingkonzepte, Strategien und Taktiken für den Unternehmer leicht machen und sie dem Unternehmer nahe bringen, sei hier ein Beispiel zum Merchandising/Vermarktung angegeben:

DEFINITION $[\ldots]$ Merchandising is the promotion of goods and/or services that are available for retail sale. Merchandising includes the determination of quantities, setting prices for goods and services, creating display designs, developing marketing strategies, and establishing discounts or coupons.

Cycles of merchandising are specific to cultures and climates. These cycles accommodate school schedules and incorporate regional and seasonal holidays as well as weather.

BREAKING DOWN 'Merchandising' Merchandising can take on different and more specific definitions in regard to different aspects of retail sales. For example, in marketing, merchandising can refer to the use of one product, image or brand to sell another product, image or brand. $^{50}$

48 https://www.namestorm.de/namensagentur

49 www.poetus.de

so http://www.investopedia.com/terms/m/marketing.asp 
Unternehmer erfahren z.B., dass in den USA der Routine-Zyklus des Retails Anfang Januar mit dem Promoting des Valentins- und St. Patricks-Tags beginnt. Dann kommen der Presidents Day, die Ostern, assoziiert mit dem guten Wetter und weiter folgen Mother's Day, Memorial Day, graduation season, Father's Day, the Fourth of July, Labor Day, Halloween, Thanksgiving and Christmas. Von 11 Anhaltspunkten berufen sich 6 auf Glauben/Religion. ${ }^{51}$ Das neue Bild des Merchandising impliziert ständiges Update und auf das Voraussehen der Konsumentenerwartung. ${ }^{52}$ Ähnlich dem Prinzip divide et impera, gibt es im Marketing die Marktsegmentierung, welche den Companies gestattet, auf unterschiedliche Gruppen abzuzielen, welche den Gesamtwert mancher Produkte und Dienstleistungen unterschiedlich voneinander wahrnehmen.

Die Segmentierung des Marktes (als Erweiterung der Marktforschung) zielt auf die Identifizierung von Konsumentengruppen $a b$, um ihnen attraktive und maßgeschneiderte Produkte und Marken anzubieten. Ziel und Zweck sind Minimierung von Risiken, Maximierung der Effizienz durch Fokussierung begrenzter Ressourcen auf das return on investment. Die Segmentierung der Märkte erfolgt nach geografischen und demografischen Kriterien (Alters-, Geschlechts-, Einkommenskriterien, Familiengröße), nach psychografischen Kriterien (sozialer Stand, life style, Persönlichkeit), nach Verhaltenskriterien. So entsteht die Möglichkeit für die Company, ihre Produkte oder Botschaften, je nach den Maßen des Marktsegments zu differenzieren. Beispiele von Marktsegmentierung findet man in den Produkten, in Marketing und Werbung, die die Verbraucher jeden Tag benutzen. Verbrauchergruppen werden mittels gezielter Produkte und Werbekampagnen angesprochen: bei älteren Personen wird, beispielsweise, bezüglich der Getreideprodukte (der Müsli - Art) ihre eigene Gesundheit erwähnt, bei junger Konsumentengruppe wird ihre Vorliebe für manche Spielfilmthemen angesprochen..$^{53}$

Namensgebung ist mittlerweile ein Beruf und ein Geschäft, denn auch die Macht des Wortes bzw. des Namens verkauft das Produkt. Professionelle Namensgeber befassen sich mit Naming und Namensfindung für Unternehmen, Marken und Produkte.

\section{Namestorm entwickelt wirkungsvolle Namen für alle geschäftlichen Bereiche:}

\footnotetext{
51 Retail Cycles in the United States http://www.investopedia.com/terms/m/marketing.

52 The New Face of Merchandising All around the world, but most notably in the United States, the reality of merchandising is getting an update. The roles and the rules of merchandising are experiencing an evolution. Chief merchants, formerly concerned mainly with selection and presentation of products, now have a broader accountability and have a heavier hand in customer experience and the development of design and talent related to display and marketing design. Because consumer savviness is broadening, and because technology plays such a massive role in merchandising, companies are feeling the need to stay ahead of consumers' expectations. Innovation and experimentation has a central role in intuitive retailers' merchandising strategies.[...] ebd.

53 Zusammengefasst nach: http://www.investopedia.com/terms/m/marketsegmenta-
} asp tion.asp 
Produktnamen, Unternehmensnamen, Markennamen sowie Namen für Webportale, Dienstleistungen und Messen Filmtitel, Buchtitel, Titel für TV-Formate, Games und Software Claims und Slogans als knackiger Zusatz für Firmen- oder Produktnamen Neben der Namensfindung für einzelne Projekte konzipieren wir auch komplette Namenssysteme für Industrie und Handel. Als ergänzende Services führen wir Marken- und Sprachrecherchen durch und erstellen auf Wunsch ein Gutachten für Ihre eigenen Namensideen. Wir machen Namen zum Eye-Catcher. ${ }^{54}$

In Blogg-Veröffentlichungen und -essayes äußern sich Blogg-Journalisten häufig zum Thema Werbung, manchmal in Verbindung mit Gottes Schöpfungen, wie zum Beispiel im Spruch Werbung ist von Gott erfunden. ${ }^{55}$

Florian Pflanz ${ }^{56}$ unternimmt mit Hilfe von theoretischen Ansätzen zu Material Religion, Populärkultur und Religionsökonomie eine theoretische Untersuchung zum Thema Marketing und Religion. Er identifiziert die vom Marketing und Branding genutzten Mittel und Wege zur „Kundengewinnung und Kundenbindung“ (S.3) und lokalisiert sie in der Contemporary Religion, um Zusammenhänge von Religion und Ökonomie $\mathrm{zu}$ erfassen: religiöse Organisationen und der globale Markt sind miteinander verknüpft und durch sozialen und kulturellen Druck wirken sie sich auf das Verhalten der Konsumenten aus. Religionswissenschaftliche Ansätze aus der Untersuchung populärkultureller Inhalte beantworten die Frage, inwieweit Religion und die Verhandlung von Religion Teil der Populärkultur sind. Diese Theorien bilden die Grundlage zum Verständnis von Branding und religionsanalogen Prozessen im Marketing von sowohl

54 https://www.namestorm.de/namensfindung

5s 04.04.08 12:38 Uhr Roland Kühl-v. Puttkamer Werbung ist von Gott erfunden Wo wir Menschen gehen und stehen, werben wir. Werbung ist nicht etwa vom Kapitalismus der Neuzeit erfunden worden. Nein, selbst Urkommunisten werben, nur anders. Wir alle werben. Natürlich könnte man Werbung auf TV-Reklame beschränken, aber dieser Ansatz wäre viel zu kurz und würde dem Werben und seinem Sinn gar nicht gerecht. Ich gehe sogar soweit: Ohne Werbung gäbe es uns alle gar nicht.Das Prinzip der menschlichen Fortpflanzung basiert ganz wesentlich auf Werbung. Menschen umwerben Menschen seit Anbeginn der Zeit, sie verführen, stellen sich in ein positives Licht, wollen Aufmerksamkeit. [...] Werbung ist Teil unseres menschlichen Miteinanders, jeden Tag. Auch im beruflichen Umfeld. Wir bewerben uns um einen Arbeitsplatz, um ein Existenzgründungsdarlehen oder den nächsten Award und stellen uns dabei möglichst in ein positives Licht. Wer eine klare Meinung vertritt, sagt: „Dafür werbe ich!“ und erhält für diesen Stil eher Zuspruch als Personen ohne Kontur und Position.Die großen Verführer waren auch immer schon die besten Werber. Sie beherrschen wie einst die erotische Zauberin bei Odysseus das subtile Bezirzen der Menschen; sie verlocken, bezaubern, entführen und reizen. Und der Verführte ist willens, ein kleines „Opfer“ zu bringen...Wir alle brauchen diese Stimulation wie die Luft zum Atmen. Wir wissen aber auch aus eigener Erfahrung und Reaktion, dass allzu plumpe Versuche das genaue Gegenteil von dem bewirken, was erreicht werden soll. Gut so! Erkennen wir diese Mechanismen, dann ist es vielleicht gar nicht so schwer, "gute“ von „schlechter“ Werbung in der Unternehmenskommunikation zu unterscheiden. 421 direkte Website-Leser 2012 Trackback-URL dieses Beitrages Schlagworte (Tags): Authentizität, verführung http://www.werbeblogger.de/2008/04/04/werbung-ist-von-gott-erfunden/

56 Marketing und Religion. Eine theoretische Untersuchung Diplomica Verlag, 2017. 
religiösen als auch säkularen Organisationen. Mit dieser theoretischen Grundlage ist es möglich, das diskursive Feld des Marketings partiell zu bearbeiten und die verschiedenen Grundlagen besser zu verstehen. ${ }^{57}$ „Im Marketing vieler säkularer Brands finden sich Rezeptionen religiöser Motive und Praktiken. [...] Rezeptionen religiöser Inhalte und Praktiken im Marketing, die in Verbindung mit sowohl religiösen als auch säkularen Produkten verbreitet werden, sind Gegenstand einer Betrachtung unter den Gesichtspunkten eines materiellen Ansatzes." ${ }^{\text {8 }}$ Aus diesem Blickwinkel, geben „konsumiertes und angenommenes Kulturgut [...] dem Produzenten soziale Macht, welche die Marktposition stärkt. Doch nicht nur die Institutionen sind in der Lage, den Markt zu beeinflussen und Macht auszuüben. Die Verbraucher bzw. Konsumenten können sich durchaus gegen institutionalisierten Druck zur Wehr setzen ${ }^{59}[. .$.$] Die$ Wechselwirkung zwischen Anbietern und Konsumenten ist der Faktor, welcher diese Macht produziert und erhält." ${ }^{30}$

\section{7. Über Benennungsgrund und -motiv}

Aus der Sicht des Benenners, ist eine Nominationseinheit „zum Zeitpunkt ihrer Prägung, stets in dem Sinn motiviert, dass er eine Zeichenkombination bildet, deren einzelne Bestandteile mit ihren Bedeutungen für ihn relevante Merkmale des Gegenstandes bzw. seines Verhältnisses zu ihm ausdrücken“, eigentlich die Definition des Okkasionalismus ${ }^{61}$. Die Aufnahme einer neuen Nominationseinheit in das Lexikon / den Namenschatz einer Sprachgemeinschaft wird dabei von den formalen und semantischen Eigenschaften gefördert oder gehemmt. ${ }^{62}$

Okkasionelle Bildungen können durch häufige Wiederverwendung in den usuellen Wortbestand einer Sprache eingehen. In viele Fällen ist es problematisch eine scharfe Abgrenzung zwischen Neologismen und Okkasionalismen vorzunehmen, es ist von einem breiten Übergangsfeld zwischen Okkasionalismen einerseits und lexikalisierten Biuldungen andererseits auszugehen. ${ }^{63}$

57 Autoren wie beispielsweise die Medienwissenschaftlerin Mara Einstein gehen in ihren Ausführungen nur wenig auf ökonomische oder popkulturelle Zusammenhänge ein. Doch nur mit diesen Bausteinen gelingt es, das Bild einer Consumer Culture zu zeichnen und zu verstehen. https://books.google.ro/books?id=yz_vDQAAQBAJ\&pg=PA3\&lpg=PA3\&dq=Gott+u $\mathrm{nd}+$ Teufel $+\mathrm{im}+$ Marketing\&source $=\mathrm{bl} \&$ ots $=\mathrm{as} 8 \mathrm{AOZ}$-bI\&sig $=\mathrm{aCpRQgunLVFqY2LIzop66zY}$ qfsg\&hl=ro\&sa $=X \& v e d=0$ ahUKEwivgKmb2_vSAhULahoKHV39AawQ6AEISzAG\#v=onep age\&q=Gott\%20und\%20Teufel\%20im\%20Marketing\&f=false

58 Pflanz, 2017, S.8-9.

59 Pflanz, 2017, S.14 ....wie der amerikanische Religionswissenschafteler Hugh Urban feststellt. // Hugh B. Urban, 2003, „Pierre Boudieu and the Study of Religion“ in: Method and Theory in the Study of Religion, 15:354 ff.

60 Pflanz, 2017, S. 14.

61 Fleischer/Barz, 1995, S.16, zitiert in Ankenbauer, 2010, S.48.

62 Ankenbauer, 2010, S.48-49.

63 Wortbildungsstrategien in der Werbung: zur Funktion und Struktur von Wortneubildungen in Printanzeigen Ulrike Krieg Buske Verlag, 2005, S.51. 
Unter Nomination versteht man „einen Akt der Nutzung eines Zeichens in der sprachlichen Handlung” ${ }^{64}$ oder "sprachliche Teilhandlung, durch die ein Sprecher einem Hörer den von ihm gemeinten Gegenstand oder Sachverhalt mittels einer bereits vorhandenen oder neu gebildeten Benennung kognitiv verfügbar macht“65 „Die Benennung einer Entität kann sehrverschieden motiviert sein, der Benenner kann einen objektiv wahrnehmbaren Aspekt der Entität beschreiben, Assoziationen zu Lebewesen oder Entitäten im Umfeld herstellen, die Entität jemandem widmen, ein Ereignis, das mit der Entität in Verbindung steht, sprachlich festhalten “66 Nomination ist „stellungbeziehende, wertende Form der Referenz unter intentional selektierender Verwendung jeweils eines bestimmten, als adäquat eingeschätzten Nominationsausdrucks [...], der aus einem Teilinventar verfügbarer Nominationsausdrücke entnommen wird “67 Nach Bellmann sei Nomination „Referenz und Pragmatik“68

Die Nominationshandlung soll drei simultane Vorgänge integrieren: 1. Der Benenner weist einer noch unbekannten Entität einen neuen Klassennamen zu; 2. Gleichzeitig weist der Benenner impliziert einer noch unbekannten Klasse diesen neuen Klassennamen zu; durch die Benennungshandlung ordnet der Benenner die Entität in diese Klasse neu ein. ${ }^{69}$

Die Nominationseinheit ist eine „Lexie oder [ein] lexikalisierbarer sprachlicher Ausdruck, die eine Entität oder eine Klasse von Entitäten unabhängig vom konkreten Sprechakt, sprachlich repräsentieren. NE [Nomnationseinheiten] unterteilen sich nach ihrer Verwendungsbreite in Eigennamen (die nur einzelne Entitäten repräsentieren) und Klassennamen (die für eine Klasse von Entitäten stehen). Formal lassen sie sich in Wörter und Phraseologismen unterteilen. ${ }^{\text {"70 }}$

Nach Fleischer ${ }^{71}$ beruht die Prägung einer neuen Nominationseinheit auf dem „Zusammenwirken semantischer (inhaltsseitiger) und formativstruktureller (ausdrucksseitiger) Prozesse“ durch Wortbildung, Phraseologismenbildung, semantische Veränderung bereits vorhandener Lexien, Transfer einer Nominationseinheit aus einer anderen Sprache und Wortschöpfung.

Ankenbauer präsentiert weiter ${ }^{72}$ die Benennungsprinzipien nach George

${ }^{64}$ Fleischer, Helbig, Lerchner 2001: 110.

65 Definition von Burkhard Schaeder im Metzler-Lexikon Sprache von Glück 2000, S. 478.

66 Norbert Ankenbauer, 2010, Das ich mochte meer newer dyng erfaren: die Versprachlichung des Neuen in den Paesi novamente retrovati (Vicenza, 1507) und in ihrer deutschen Übersetzung (Nürnberg, 1508), Frank \& Timme GmbH, S.16.

67 Bellmann, 1989, S.30 in Akenbauer, 2010, S.23.

68 Bellmann, 1989, S.31 in Akenbauer, 2010, S.23.

69 Die Definition von Wiegand,1996, S.72, beschreibt die obigen 3 Vorgänge in: Ankenbauer, 2010, S. 25-26.

70 Ankenbauer, 2010, S. 28.

71 Fleischer, 1989, S.14.

72 Ankenbauer, 2010, S.33-38. 
R. Stewart ${ }^{73}$, aufgestellt in Verbindung mit Toponymen, allerdings gelten sie für einen breiteren Kreis von Benennungen.

1. Prinzip von Entität (abgrenzbar) und Nutzen (der Bezug daruf soll nützlich sein). 2. Prinzip des genusindizierenden (was ist das? Also die Klasse.) und des spezifizierenden Elements (was grenzt die Entität von anderen derselben Klasse ab oder der spezifische Unterschied) 3. Prinzip des Außergewöhnlichen: der Benenner bezieht sich auf etwas Besonderes 4. Prinzip des spezifischen Wortschatzes und der Wiederholung (genusindizierende und spezifizierende Elemente die sich wiederholen) 5. Prinzip der Analogie (im Fall der Toponyme, solche die an andere formalähnlich angleichen) 6. Prinzip von Gegenstück und Systemhaftigkeit (im Fall der Toponyme, spezifizierende Bestandteile, die einen Bezug zu weiteren Toponymen herstellen, z.B. Insel Christus, dann Maria, dann Namen der spanischen Königsfamilie) 7. Prinzip des Standpunktes des Benenners (Nord, Süd) 8. Prinzip des dualen und multiplen Ursprungs (mehrere Motivationen / eine historische und eine persönliche) 9. Prinzip des fossilen genusindizierenden Elements (andere Sprachen oder ältere Sprachstufen, wobei das spezifizierende Element ein verdunkeltes genusindizierendes Element aus einer anderen Sprache ist.

Nach Stewart ${ }^{74}$ gäbe es eine Vielzahl von Benennungstypen, die er am Beispiel von Toponymen identifizert hat:

- deskriptive Namen, mit ihren Unterklassen: n. sensorisch wahrnehmbaren Eigenschaften;
n. relativen Eigenschaften in Bezug zu einer anderen Eigenschaft: Nordsee/Südsee, Altstadt/
Neustadt, intelektuelle Eigenschaften (Ecuador), metaphorische Namen: eine Eigenschaft ist
eine Metapher; n. subjektivem Eindruck; negativ/ironische Namen; mahnende Namen; n.
regelmäßigen Erscheinungen benannte Entitäten; -assoziative Namen (in Verbindung mit
anderen Entitäten); -Ereignisnamen (Naturereignis, Kalendernamen; Ereignisse mit Tieren,
Gefühle, Menschen); -Besitzanzeigende Namen; - Kommemorative Namen; - Werbende, einneh-
mende Namen (was sein soll/könnte, attraktiv machende Namen (Kap der Guten Hoffnung);
-Volksetymologien; -Neu geschaffene Namen (Wörtschöpfung); - Fehlerhafte Namen; - Überge-
gangene/übertragene (Isola Rossa, nach Capo Rosso)

Clemens-Peter Herbermann ${ }^{75}$ stellt 18 Benennungsprinzipien auf, welche nicht nur für Toponyme, sondern für die Namensgebung im Allgemeinen gültig sein soll.

- Technologisches Prinzip: Gehacktes, Aufschnitt, Schlagsahne auch Tasteninstrument, Dampfschiff; -Teleologisches Prinzip, d.h. zweckorientiert - wofür/wogegen: Suppenlöffel, Wecker; - hylologisches Prinzip, nach dem Stoff, Material: Roggenbrot, Stahlfeder; - topologisches Prinzip, nach Ort / Zeit, wo sich das Denotat befindet/stattfindet: Turmuhr, Nachtmarsch; - phänomenologisches Prinzip, nach Farbe, Form, Größe, akustischen, gustatorischen, olfaktorischen, haptischen Eigenschaften, nach Konsistenz;

73 Stewart, George R. 1975. Names on the Globe. New York: Oxford University Press.

74 Stewart, 1975, in Ankenbauer 2010, S.39.

75 Herbermann, 1998, S. 70-91 in Ankenbauer, 2010, S.45-47. 
- aitiologisches Prinzip, nach der Ursache: Brandmal, Freudentränen; - praxeologischesethologisches Prinzip, nach Verhalten, Handlung des Denotats: Genießer, Bäcker, Kletterrose; - trophologisches Prinzip, nach der Nahrung des Denotats: Aasgeier, Kartoffelkäfer; - konsumentenbezogenes Prinzip, nach dem Verbraucher des Denotats: Bauernfrühstück, Gänseblümchen; - nach dem Urheber, Erfinder, Entdecker: Ottomotor; - Benennung nach dem das Denotat Realisierenden: Zigeunermusik; - Nach dem Adressaten/Rezipienten des Denotats: Jugendliteratur; - Nach dem vom Denotat Betroffenen: Kinderlähmung; - Symptomatologische Benennung: Schlafkrankheit; - Nach der Bedingung der Ausführung des Denotats: Hürdenlauf, Gepäckmarsch; - Nach dem Behälter, Aufbewahrungsart des Denotats: Dosenmilch, Beuteltee; - Nach der Art der Wertschätzung des Denotats: Schundliteratur.

\section{Zur Nomination in der Kochsprache}

Konventionelle und nicht konventionelle Träger der Kochsprache mit ihren kulinarischen / gastronomischen Termini / Halbtermini (Kochbücher, -lexika, und -zeitschriften, Werbezeitschriften und -flugblätter, Menu- und Zutatenvorschläge, Rezepte auf Koch-Sites und -Bloggs im Internet, Werbeplakate und elektronische Werbeschirme im öffentlichen Raum u.v.a.) kommen an den Markt, wenden sich an den Kunden und manipulieren ihn denn Der Wurm muss dem Fisch schmecken, nicht dem Angler.

Die kulinarischen Bezeichnungen sind vielfach motiviert; Speisenamen sind Nominationseinheiten, d.h. „sprachliche Ausdrücke, die einen Wirklichkeitsausschnitt als ,Gegenstand" repräsentieren"76 und sind als Produkte der Nominationsbildung zu verstehen, d.h. „der Prägung (Schaffung) oder ,Herausbildung' (Entwicklung) einer neuen Nominationseinheit"77. Zur Charakteristik des kulinarischen Wortschatzes bzw. zur Stellung der kulinarischen Lexik im Wortschatz der jeweiligen Sprache bemerkt Turska ${ }^{78}$, dass die Abgrenzung der Fachsprache von der Allgemeinsprache schwierig und die Grenze fließend ist. Kochsprache ist für Turska „ein Sonderfall an sich”. Drei allgemeingültige Feststellungen unterstützen ihre Behauptung: 1. man muss kein Fachmann sein, um sich über die Küche zu unterhalten. 2. jeder besitzt Grundkenntnisse im Bereich des Kochens. 3. die Kochsprache wie andere Fachsprachen könnte ohne den Grundwortschatz nicht existieren ${ }^{79}$.

Hier weiter ein auf Grund von Internetrecherchen und Gesprächen mit Informanten angelegtes Korpus zu Namen von Lebens- und Genussmitteln und komplexeren Speisenamen in Verbindung mit heilig und profan, göttlich und teuflisch in der Domäne Essen/Trinken/Gastronomie, wie auch einige Bezeichnungen von Heilkräutern.

\section{Gott, Jesus Christus}

\footnotetext{
76 Fleischer 1989: 13.

77 Fleischer 1989: 14.

78 Turska, Marta. 2009. Internationalismen in der Fachsprache der Gastronomie und Kochkunst im fünfsprachigen Vergleich. Peter Lang.

79 Vgl. Turska, 2009, S.16-17.
} 


\begin{tabular}{|l|l|}
\hline Christstollen & Weihnachtskuchen \\
\hline Williams Christ Birnen / pere vilmoșe & $\begin{array}{l}\text { Williams Christ: eine Sorte der Birne (Pyrus commu- } \\
\text { nis) }\end{array}$ \\
\hline Vin "Via Domnului" & Gottes Weingarten, rum. Wein \\
\hline Ah-mon-Dieu & Birnensorte \\
\hline
\end{tabular}

${ }^{7}$ Auch Obstbrand. Ursprüngl. Name: Bon- 1770 nach England gelangt sein und dann von Chrétien Williams [USA u. Kanada: Bartlett]. der Baumschule Williams in ganz GroßbritanÄlteste Nachweise: etwa 1770 (England) nien verbreitet worden sein. Mitte des 19.Jahrzurück. Benannt nach ihrem ersten Verbreiter, hunderts war diese Sorte in Belgien bekannter dem Baumschuler Williams aus London. Der als in England und fand damals über den belBeiname: Bon-Chrétien frz. „Guter Christ“. gischen Pomologen van Mons Verbreitung in Dieser soll sich auf den Heiligen Franz von ganz Europa sowie in Nord- und Südamerika. Paola beziehen, der dem französischen König In den 1920er Jahren war die Williams Christ Ludwig XI., genannt der Kluge, Birnensa- eine der drei Birnensorten, die zu Reichsobstmen aus Kalabrien angeboten hat, die dann sorten gewählt wurden. Abgek. nach https:// auf königlichen Befehl ausgesamt wurden. de.wikipedia.org/wiki/Williams_Christ Abkömmlinge dieser Varietät sollen dann um

\section{Engel, Himmel}

Angelli (Engel, it. Getränkemarke); Vin 9 ceruri (9 Himmel Handelsname eines rum. Weines); Blonder Engel

(Orangensaft, Eierlikör; Eis) $^{8}$; Engelskuchen; Engel zu Pferde'; Engelskuss (Dessert mit Mascarpone und Quark $)^{10}$

${ }^{8}$ https://www.chefkoch.de/forum/2,22,25393/Sot-l-y-jette.html

${ }^{9}$ https://www.chefkoch.de/forum/2,22,25393/Sot-l-y-jette.html?page=2

${ }^{10} \mathrm{https}$ ///www.chefkoch.de/rezepte/2168861348038269/Andis-Engelskuss.html

\section{Heilige und biblische Gestalten}

Ambrosiuscreme; Valentinskuchen; Frischlingskeule Sankt Hubertus; Johannisbrot; Martins Gans; coquilles Saint-Jacques; vin saint-honoré, vin saint-émilion, vin saint-nectaire (Au repas de ce soir, nous avions un excellent saint-émilion qui accompagnait un délicieux saint-nectaire. En dessert, le saint-honoré était l'apothéose de ce dîner de fête. ${ }^{11}$ ); pecetea lui Solomon (polygonatum odoratum ${ }^{12}$ )

${ }^{11}$ https://www.francaisfacile.com/exercices/exercice-francais-2/exercice-francais-51961.php

${ }^{12} \mathrm{http}: / /$ www.sfatulmedicului.ro/plante-medicinale/pecetea-lui-solomon-polygonatum-odoratum_14439

\section{Teufel}

Diabolo-menthe; Sauce diable / Teufelsosse; Poulet a la diable (Hähnchen a la diable); Pizza diavolo; Sacla Arrabbiata, sauce italienne aux tomates à la diable ${ }^{13}$; Palette à la diable à l'alsacienne $^{14}$; Pomme de terre à la diable ${ }^{15}$; Huîtres à la diable ${ }^{16}$; Oeufs à La Diable $e^{17}$ / Teufelseier; Spaghetti tomate et piment à la diable ${ }^{18}$; Fra Diavolo Sauce ${ }^{19}$; Teufelsküsse (Weihnachtsplätzchen) ${ }^{20}$

\footnotetext{
${ }^{13}$ https://www.monoprix.fr/arrabbiata- ${ }^{15}$ recette-pomme-de-terre.com/.../pomme-
} sauce-italie... de-terre...

${ }^{14}$ www.cuisineactuelle.fr $>\ldots$ P Plat principal 
${ }^{16}$ www.elle.fr > Elle à Table > Recettes de cuisine

${ }^{17}$ https://www.frenchs.ca/.../oeufs--la-diable-frenc...

${ }^{18}$ www.fourchette-et-bikini.fr > Recettes minceur
${ }^{19}$ https://www.pinterest.de/explore/saucediavolo-rezept/

${ }^{20}$ https : / / www.chefkoch.de / rezepte/2075211335382238/Teufelskuesse. html

29 Teufel-Rezepte: Teufels Miesmuscheln; ,Teufels Art' Möhrensuppe; Marinierte Teufels - Garnelen; Armer Teufel Spaghetti; Des Teufels Leibgericht; Teufels-Thunfischmakkaroni; Teufel - Tomatensoße; Tomaten des Teufels; Teufels Wurstsalat; Teufel - Dip all'italiamann; Teufels Pute; Teufels - Sauce; Teufels-Currysoße zur Currywurst; Grüner Teufel; Teufels - Nudelsalat; Little Devil (kleiner Teufel); Teufels - Polenta; Teufel; Teufel - Salat; Teufels - Kuchen; TeufelsSteaksauce; Teufels-Ragout; SABO Teufels Hähnchen ${ }^{21}$

\section{Mönche, Nonnen, Klöster, Vatikan}

\begin{tabular}{|c|c|}
\hline Cappuccino & $\begin{array}{l}\text { It. cappuccino, zu: cappuccio = Kapuze, nach der Farbe der Kutte, die } \\
\text { von den Kapuzinermönchen getragen wird }\end{array}$ \\
\hline Gnocchi al Vaticano & $\begin{array}{l}\text { The Vatican Cookbook: Presented by the Pontifical Swiss Guard: } 500 \text { Years } \\
\text { of Classic Recipes, Papal Tributes, \& Exclusive Images, The Pontifical } \\
\text { Swiss Guard, Sophia Institute Press, } 5 \text { apr. } 2016-204 \text { p. }^{22}\end{array}$ \\
\hline Abteilikör & $\begin{array}{l}\text { mit Kräutern, Honig u. a. hergestellter, meist kräftiger, goldgelber Likör. } \\
\text { Der Likör geht auf alte Klosterrezepte zurück }\end{array}$ \\
\hline Sfintisori, Mucenici & « kleine Heiligen". \\
\hline Krampus & $\begin{array}{l}\text { Der Krampus ist im Brauchtum eine Schreckgestalt des Adventsbrauch- } \\
\text { tums, in Begleitung des Hl. Nikolaus. (Verbreitung: im Ostalpenraum, } \\
\text { südlichen Bayern u. der Oberpfalz, in Österreich, Teilen des Fürsten- } \\
\text { tums Liechtenstein, in Ungarn, Slowenien, der Slowakei, in Tschechien, } \\
\text { Südtirol, Welschtirol (Trentino), Teilen des außeralpinen Norditaliens } \\
\text { und Teilen Kroatiens. Während der Nikolaus die braven Kinder be- } \\
\text { schenkt, werden die unartigen vom Krampus bestraft. Die Gestalt des } \\
\text { Krampus stammt ursprünglich aus der vorchristlichen Zeit. }\end{array}$ \\
\hline Vin mânăstiresc & $\begin{array}{l}\text { Vom Klosterweinbau stammendee Weine; Sammelname, Handelsname } \\
\text { eines Weines }\end{array}$ \\
\hline Vin bisericesc & $\begin{array}{l}\text { Im Kirchenritual verwendeter Wein; Sammelname, Handelsname eines } \\
\text { rum.Weines }\end{array}$ \\
\hline Bucate mânăstirești & Rum. Bezeichnung für im Kloster übliche Speisen \\
\hline Pui mânăstiresc & Rum. Kochrezept, „Hähnchen Klosterart“ \\
\hline Șoapta călugărului & Rum. Wein „Des Mönchs Geflüster” \\
\hline Le jésuite & $\begin{array}{l}\text { Un triangle de pâte feuilletée garni de frangipane et recouvert d'amandes ou } \\
\text { d'un glaçage. A l'origine, le généreux glaçage formait un dôme et la pâtisserie } \\
\text { ressemblait alors au chapeau à bords relevés porté par les Jésuites. }{ }^{24}\end{array}$ \\
\hline Le boule des moines & $\begin{array}{l}\text { Un fromage frais au lait de vache cru, arômatisé à l'ail, ciboulette et au poivre. } \\
\text { Il est originaire du Morvan. Depuis 1920, ce fromage était fabriqué par les } \\
\text { moines bénédictins de l'abbaye de Sainte-Marie-de-la-Pierre-qui-Vire. La fa- } \\
\text { brication et l'exploitation a été confiée à des agriculteurs proches de l'abbaye } \\
\text { à partir de } 1988 .{ }^{25}\end{array}$ \\
\hline
\end{tabular}




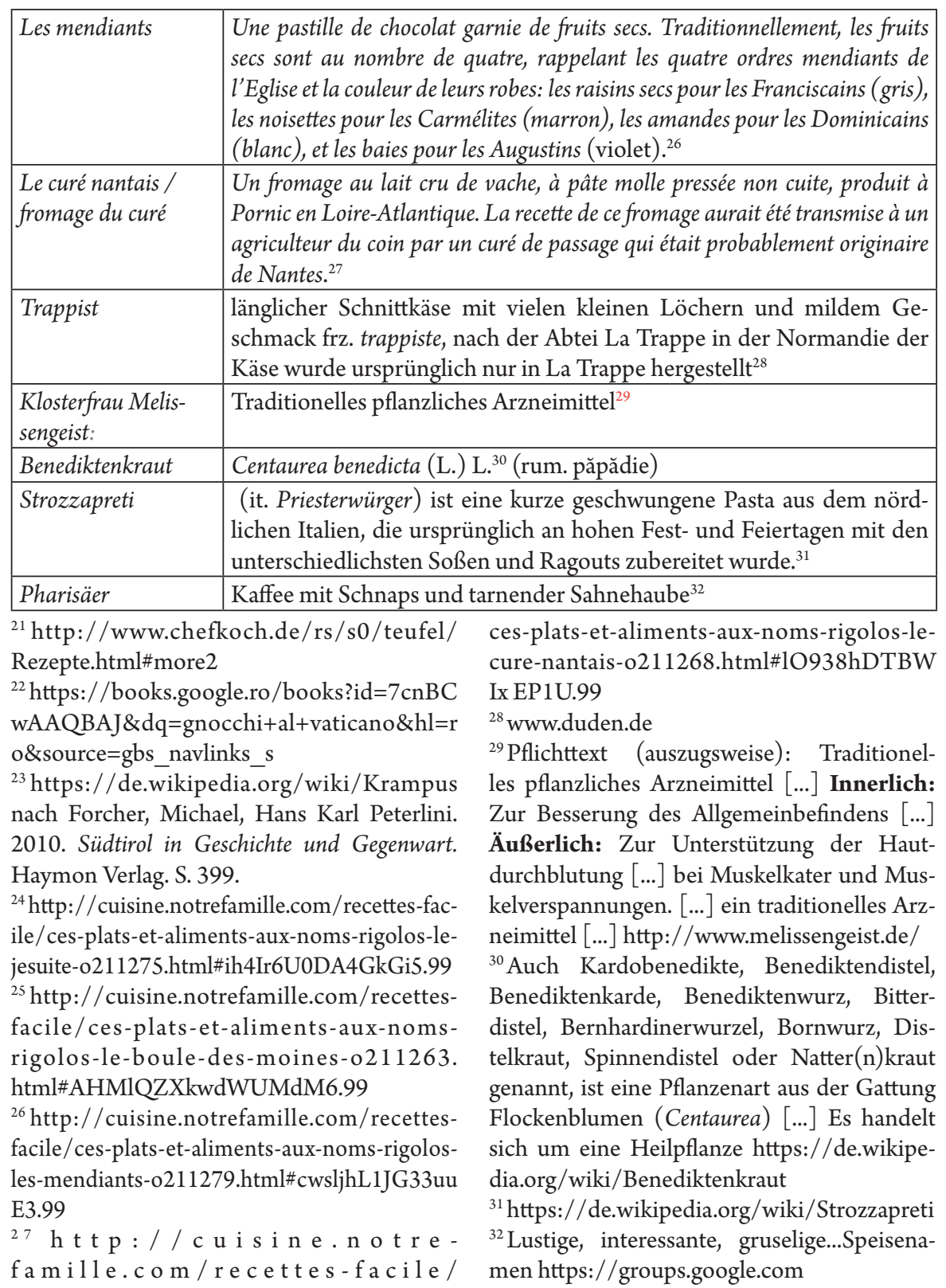




\title{
Andere: Kapuzinerkoch / Besoffener Kapuziner ${ }^{80}$ Durstige Nonne ${ }^{81}$ Bischofsbrot; Kardinal; Ohnmächtiger Priester; Pfaffenhütchen ${ }^{82}$
}

\section{Religiöse Feiertage}

\author{
Weihnachtsstollen; Pasca, paska, pessach Brot (Osterkuchen/Brot)
}

\section{Vorchristliche mythologische Gestalten}

Sânziene ${ }^{33}$; Götterspeise a.<ohne Plural> Ambrosia (1) b.(scherzhaft) köstliche Speise aus Gelatine mit Aromastoffen oder unter Verwendung von Fruchtsaft hergestellte Süßspeise ${ }^{34}$; Feenspeise $^{35}$; Hexenschnee ${ }^{36}$; Gebackene Hexenbesen ${ }^{37}$; Gespenstergrütze ${ }^{38}$; Jeisterspucke [Geisterspucke] (Aal in Aspik) ${ }^{39}$

${ }^{33}$ Galium mollugo www.sanatatecuplante.ro/ plante-medicinale/plante...d/136-dragaicasanzienele.html Erlen; rum. ielele, dânsele, drăgaice, vâlve (vâlva băii, vâlva pădurii), irodițe, rusalii, nagode, vântoase, domnițe, măiestre, frumoase, împărătesele-văzduhului, fetelecodrului, fetele-câmpului, șoimane, mușate, miluite, albe, [...] sânzienele, gute Feen. Gem. dem Historiker Vasile Pârvan und Mircea Eliade: von San(cta) Diana, röm. Jagd- und Waldgöttin; [...] Heil- und Speisepflanze [...] ${ }^{34}$ http://www.duden.de/rechtschreibung/ Goetterspeise

${ }^{35}$ Vanillecreme mit geknusperten Flakes und Gewürzkirschen http://www.kruse-hollotal. de/index.php/speisekarte.html

36Eiklar steif aufschlagen. nach und nach Zucker reinrieseln. Saft einer Zitrone und Rum dazugeben. Apfelpüree locker unterziehen. In einer Schale oder vier Gläser befüllen. Kleine Johannisbeertupfer draufsetzen.

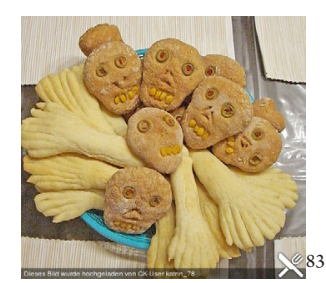

Gebackene Hexenbesen
Geriebene Schoko drübersteuen. Sofort zu Tisch bringen. https://www.ichkoche.at/ hexenschnee-rezept-22353

${ }^{37}$ Lustige_Namen www.chefkoch.de

${ }^{38} 1$ [...] Johannisbeeren waschen, abtropfen lassen und die Beeren [...] von den Rispen befreien. Kirschen waschen und entsteinen. Himbeeren verlesen. 2 Speisestärke mit 6 EL von dem Kirschsaft anrühren. Den restl. Kirschsaft mit Zucker zum Kochen bringen. Die angerührte Speisestärke unterrühren und einmal aufkochen lassen. Topf vom Herd nehmen und die vorbereiteten Früchte unterrrühren. Die Grütze in eine Schale füllen und kalt stellen. 3 Für die Sauce aus Pulver, Milch, Zucker und Vanillezucker nach Anleitung eine Sauce zubereiten. 4 Die Gespenstergrütze mit der Vanillesauce (mehr dazu bei www.kochbar.de)

${ }^{39}$ Lustige, interessante, gruselige...Speisenamen https://groups.google.com

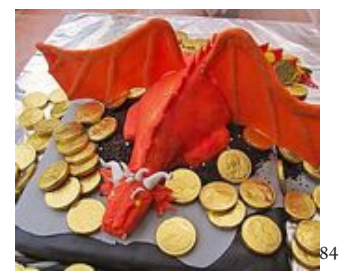

Liebe Sünde, Schokoladenkuchen

\footnotetext{
80 https://www.chefkoch.de/forum/2,22,25393/Sot-l-y-jette.html?page=3

81 https://www.chefkoch.de/forum/2,1,96351/Ich-Suche-Rezepte-mit-witzigen-Namen.html

82 https://www.chefkoch.de/forum/2,22,25393/Sot-l-y-jette.html?page=2

83 https://ro.pinterest.com/pin/441282463467031459/

${ }^{84}$ https://www.chefkoch.de/rs/s0/liebe+s\%C3\%BCnde/Rezepte.html
} 


\section{Kirchliches Geschirr, andere Artefakte}

rum. Crucea voinicului "Des Rüstigen Kreuz" ${ }^{40}$; rum. Cădelnița "Weihrauchfass" ${ }^{41}$; rum. clopoțele / iarba Sfântului Gheorghe „Skt. Georgs Glöckchen“ / auch Gras ${ }^{42}$; rum. Trompeta ingerilor „Trompete der Engel“43 / Trompeta-ingerului „Engelstrompete“44 /Trompeta-ingerului „Engelstrompete“ 45

${ }^{40}$ Hepatica transsilvanica http://infozone. forumhit.ro/t364-denumirea-stintifiica-aplantelor

${ }^{41}$ Campanula carpatica http://infozone.forumhit.ro/t364-denumirea-stintifica-a-plantelor ${ }^{42}$ Dt.Maiglöckchen,rum.lăcrămioara (lat.Convallaria majalis); http://www.csid.ro/plantemedicinale-fitoterapice-si-gemoterapice/ lacramioara-convallaria-majalis-11835520/
${ }^{43}$ Brugmansia arborea sin. Datura arborea. www.horticultorul.ro/flori-de-apartament.../ trompeta-ingerilor-brugmansia-arborea/

${ }^{44}$ Brugmansia sanguinea https://bodygeek.ro $>$ Naturist \& Plante medicinale

${ }^{45}$ Brugmansia aurea www.eplante.ro > Plante $\mathrm{AZ}>$ Litera B

\section{Organe / Teufel, Engel, Mutter Gottes, Mönche, Nonnen, physiologische Prozesse} rum. limba diavolului / ung. ördögnyelv „Teufelszunge“46; rum. ghiara diavolului „Teufelskralle“47; rum. puța îngerului / puțișoare, ung. angyalbögyörö „Engelspenis“, wie Gnocchi, aber als Dessert, mit Zucker und Nuss; dt. Mönchskopf / frz. tête de moine; rum. palma Maicii Domnului ${ }^{48}$ Salbei „Mutter Gottes Handfläche”; rum. mâna Maicii Domnului ${ }^{49}$ Efeu „Mutter Gottes Hand"; dt. Nonnenfürzle / frz. pets de nonne

${ }^{46}$ glucomanan de konjac / capsule http:// sanatoasa/remedii-naturale/plantele-de-leacadiograsime.ro/magazin/promotii-actuale/ capsule-glucomanan-konjac-detalii.html ale-maicii-domnului-1184327

${ }^{49}$ Caprifoiul (Lonicera japonica), https:// ${ }^{47}$ Harpagophytum procumbens www.csid.ro/ plante...si.../gheara-diavolului-harpagophytum-procumbens-11447535/

${ }^{48}$ Salvia; $\quad$ http://www.ziare.com/viatawww.realitatea.net/ce-beneficii-are-manamaicii-domnului-pentru-sanatate_1712336. html\#sthash.Hn4pZvux.dpuf

\section{Ethnisch-geografische Herkunft, Zweck / biblisch, religiös, abgrenzend}

rum. Mana cereasca (Himmlische Mannah); rum. Pasca jidoveasca (Jüdisches Osterkuchen/ Brot); rum. miel de pasti (Osterlamm)

\section{Himmel und Hölle ${ }^{85}$}

85 Cremig vs. scharf Himmel und Hölle ist ein beliebtes Motto für Feiern oder Dinnerparties. Besonders stilecht ist es mit passendem Essen, das vom Spiel mit Kontrasten lebt. Zum Thema Himmel passt helles Essen mit einer luftigen Konsistenz. Der erste Gang besteht aus aufgeschäumter Kartoffelsuppe oder Petersilienwurzelschaum mit Flusskrebsen. Pasta mit Sahnesauce, Gratins aller Art oder Schnitzel im Kokosmantel sind leckere Hauptgerichte. Als Dessert schmecken Torten und Cremes mit Mascarpone, weißer Schokolade und Vanille. [...] Für das Motto Hölle konzentrieren Sie sich auf rote, schwarze und natürlich pikante Lebensmittel. Beginnen Sie das Dinner mit einer Tomatensuppe und servieren Sie als Hauptgang brasilianische Feijoada aus schwarzen Bohnen. Auch Tinentenfisch-Pasta ist ein richtiger Hingucker und hat zudem ein feines Aroma. Als Dessert reichen Sie Süßspeisen mit 
Tortellini paradiso e inferno e rapidamente / Tortellini Himmel und Hölle und turboschnell ${ }^{50}$; Bucatini all amatriciana oder Nudeln zwischen Himmel und Hölle (Quelle: www.kochbar. de $)^{51}$; Hähnchenschenkel Himmel und Hölle ${ }^{52}$; Dessert - Himmel und Hölle ${ }^{53}$; Himmel und Hölle [Hauptgericht ${ }^{54}$; Fleisch - Himmel und Hölle a'la Manfred ${ }^{55}$; Himmel und Ä̈̈d [Erde] (gebratene Blutwurst mit Apfel/Kartoffeln) und Schlesisches Himmelreich (Kartoffelklößer mit Backobst und Bauchspeck $)^{56}$

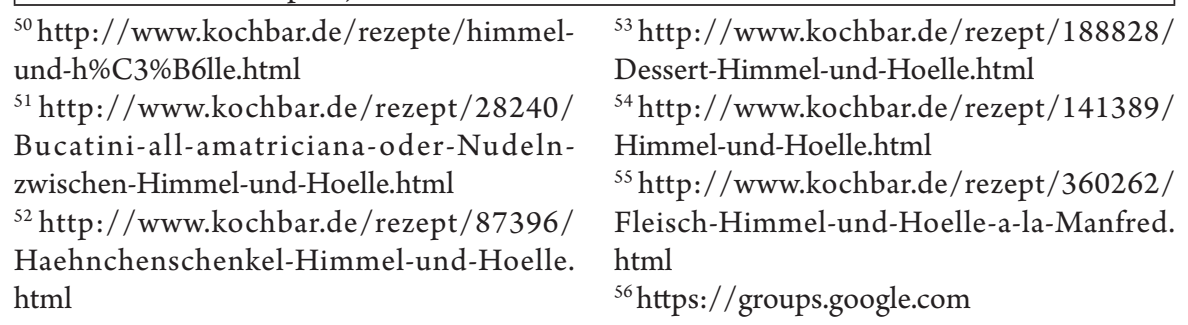

Sünde

Liebe Sünde $^{57}$, Süße Sünde ${ }^{58}$, Kalte Sünde - Heiße Liebe s9 $^{59}$

${ }^{57}$ https://www.chefkoch.de/rs / s 0 / 1145131220866952/Suesse-Suende-Dessert. liebe+s\%C3\%BCnde/Rezepte.html html

${ }^{58}$ https://www.chefkoch.de/rezepte/ $\quad{ }^{59}$ https://www.kochbar.de/rezept/199982/ Kalte-Suende-Heisse-Liebe.html

Was dem obigen Korpus abzulesen sei: Sprache kennt keine Scham / Schande, wie der rumänische Sprachwissenschaftler Alexandru Graur ${ }^{86}$ zu sagen pflegte; erst im Sprachgebrauch, in den Bedingungen interpersonaler bzw. sozialer Beziehungen der Kommunikation, tritt diese soziale Emotion auf; in seiner individuellen, intentionalen Handlung scheut der anonyme Namensgeber weder den Teufel, noch fürchtet er sich vor Gott, oder dass er „blasphemisch“ vorgehen würde, wenn er Dingen Namen gibt. Der Namensgeber handelt aufrichtig, kreativ oder humorvoll / witzig / spöttisch (dt. Nonnenfürzle / frz. pets de nonne), symbolisch, metaphorisch, komparativ (rum. Trompeta-ingerului / dt. Engelstrompete), mit großer Genauigkeit und nach gründlicher Beobachtung des zu nennenden Objektes bzw. seiner Form (-hütchen, -trompete), seiner Farbe (Les mendiants); seiner Geräusche (Nonnenfürzle), seines Verhaltens und körperlich-psychischen Zustandes (Durstige Nonne; Ohnmächtiger Priester). Er nennt etwas infolge eines Vergleichs mit anderen ihm bekannten Gegenständen (rum. cădelniţa das Weihrauchgefäß; crucea das Kreuz), aber bestimmt, schämt er sich seines Wortgebrauchs nicht (puţa îngerului „Engelspenis“ dt. Bubespitzle bzw. Schupfnudeln ${ }^{87}$ ) und ebenfalls achtet auf keine Moral.

dunkler Schokolade, Erdbeeren, Himbeeren und Chili.[...] http://www.kochbar.de/rezepte/himmel-und-h\%C3\%B6lle.html

86 Lebensjahre: 1900-1988; Mitglied der Rumänischen Akademie;

87 Zipfel, Wichspfeiferl; oder im Sudetenland: Mohnschlischgern; oder in Schwaben 


\section{Gott und der Teufel, Himmel und Hölle, heilig und profan, komplementär}

Laut zahlreichen Sprichwörtern, sind manche Handlungen rund um menschliche Beschäftigungen wie bauen, schaffen, kochen, aber auch um Glaube selbst, Ergebnis einer Art antithetischen team works von Gott und dem Teufel, zu einem komplementär gestalteten Zweck, zu verzeichnen. Es geht nicht um das Gute und das Böse, sondern um zusammenhängende Handlungen oder Begriffe, die assoziiert werden. Einen antithetischen Unterschied zwischen den Beiträgen des Teams Gott -Teufel am gemeinsamen Werk gibt es trotzdem: wenn beide „Partner“ bauen, ist das Objekt des Teufels kleiner; Gott stiftet, der Teufel baut; Gott bringt die Mittel, die Energie (Feuer, Lebensmittel), der Teufel nur die Verpackung und die Arbeit.

\begin{tabular}{|l|l|l|l|}
\hline Sprichwörter & Objekt - Handlung & Gott & Teufel \\
\hline $\begin{array}{l}\text { Wo Gott eine Kirche stiftet, baut der Teufel eine } \\
\text { Kapelle. }\end{array}$ & $\begin{array}{l}\text { Objekt: wird gestiftet vs. } \\
\text { wird gebaut; groß vs. klein }\end{array}$ & Kirche & Kapelle \\
\hline $\begin{array}{l}\text { Als Gott das Feuer schuf, schuf der Teufel den } \\
\text { Kessel. }\end{array}$ & Energie - Behälter: & Feuer & Kessel \\
\hline $\begin{array}{l}\text { Gott schickt ihm [dem Menschen] wohl die } \\
\text { Speisen, aber der Teufel kocht sie. }\end{array}$ & Lebensmittel - Zubereitung & Speisen & kochen \\
\hline $\begin{array}{l}\text { Wenn Gott uns Mehl gibt, bindet der Teufel } \\
\text { den Sack zu / nimmt der Teufel de Sack weg. }\end{array}$ & Lebensmittel - Verpackung & Mehl & Sack \\
\hline $\begin{array}{l}\text { Gibt Gott Fleisch, so will's der Teufel braten } \\
\text { (kochen). Engl.: God sends meat and the } \\
\text { devil sends cooks. }(\text { Bohn II, 81.) }\end{array}$ & Lebensmittel - Zubereitung & Fleisch & kochen \\
\hline
\end{tabular}

${ }^{60}$ Sprichwörter der germanischen und Gott 637, S. 330. http://www.bauernregeln. romanischen Sprachen vergleichend zusam- net/kirche.html; http://www.bauernregeln. mengestellt von Ida von Düringsfeld und net/essen.html

Otto Freiherrn von Reinsberg-Düringsfeld, ${ }^{61} \mathrm{http}$ ://www.zeno.org/Wander-1867/A/ Leipzig. Verlag voon Hermann Fries 1872, Gott (Artikel 207)

Für äußerst kreative Benennung hat der anonyme oder professionelle Namenfinder / Namensgeber, auf der Gourmetmeile Minden ${ }^{88}$ jedenfalls gesorgt. Man könnte glauben, in Teufels Küche gekommen zu sein, was allerdings „in große Verlegenheit oder Gefahr geraten" ${ }^{\text {"89 }}$, bedeutet, was auch nicht weit entfernt von der

Buabasteckerl. Quelle: Lustige Speisenamen: Dumme Nuss, Tote Tante, Liwanzen. Heinz Scholz, Wissenschaftspublizist, Schopfheim, D. www.textatelier.com

88 Gourmetmeile Minden - auch Veggies werden satt, irgendwie... : Veröffentlicht am $\underline{12}$. Juli 2011 https://missglueckt.wordpress.com/2011/07/12/gourmetmeile-minden-veggie/ Quellenangabe: http://www.minden-erleben.de - Minden Marketing GmbH.

${ }^{89}$ Nach mittelalterlichem Volksglauben hatte der Teufel eine Küche, in der die Hexen (daher $>$ Hexenküche<; vgl. >Faust $<$ I) und Zauberer am Werke waren. Auch die Hölle hieß im 16. Jahrhundert $>$ des Teufels Küche $<$. In Fischarts $>$ Brotkorb $<(1584)$ heißt es: $\gg$ S. Patricius Loch, darin der Teufel ist Koch $\ll$. Verwandt ist die Redensart >In des Teufels Badstube kommen $<$, in die höchste Not geraten; vgl. schon mittelhochdeutsch > ze helle baden < (Thomasin). Von der Volkssage werden oft Teufelsbäder und Teufelsküchen genannt. In der folgenden Gruppe von 
Wahrheit ist: man ist mit vereinten himmlischen und höllischen Kräften verführt, der Esslust freie Bahn zu geben. Nicht nur der Bauchmensch wird angesprochen, sondern vielmehr geht es hier um die Fähigkeit der Namen Neugier und Interesse beim Kunden zu erwecken und spannende kulinarische Ereignisse ankünden.

\begin{tabular}{|c|c|}
\hline Name des Gerichtes & Was das ist... \\
\hline $\begin{array}{l}\text { „Himmel trifft Hölle“ (Mindener } \\
\text { Feinkosthaus) }\end{array}$ & $\begin{array}{l}\text { Pikante und milde Käsesorten mit Portwein-Feigen } \\
\text { und Cassis-Senf aus dem Klostergarten }\end{array}$ \\
\hline $\begin{array}{l}\text { "Himmlische Schweinereien“ (Min- } \\
\text { dener Feinkosthaus) }\end{array}$ & $\begin{array}{l}\text { Luftgetrockneter italenischer San Leo und spani- } \\
\text { scher Trevelez Schinken mit Winzer Brot }\end{array}$ \\
\hline "Jakobsweg" (Mindener Feinkosthaus) & $\begin{array}{l}\text { gebratene Jakobsmuscheln in einer Limetten-Kräu- } \\
\text { ter-Marinade mit Pilgerbrot \& Dipp }\end{array}$ \\
\hline $\begin{array}{l}\text { Teuflisch gute Scampis (Der Schlem- } \\
\text { merservice) }\end{array}$ & $\begin{array}{l}\text { in Basilikum-Knoblauch-Öl gebraten mit Baguette- } \\
\text { Scheiben, Aioli und Salatbeilage }\end{array}$ \\
\hline $\begin{array}{l}\text { Luzifers Leibgericht (Der Schlem- } \\
\text { merservice) }\end{array}$ & $\begin{array}{l}\text { Schweinefiletmedaillons mit französischer Pfeffer- } \\
\text { sauce, dazu kleine Kartöffelchen mit grobem Meer- } \\
\text { salz und Dipp }\end{array}$ \\
\hline $\begin{array}{l}\text { Höllisch scharf (Der Schlemmerser- } \\
\text { vice) }\end{array}$ & $\begin{array}{l}\text { Lammragout in Kokos-Currysauce mit Langkorn- } \\
\text { reis an Salatbeilage }\end{array}$ \\
\hline $\begin{array}{l}\text { Himmlischer Genuss (Dalmatien Res- } \\
\text { taurant) }\end{array}$ & $\begin{array}{l}\text { Genuss Geräucherter dalmatinischer Schinken, } \\
\text { Schafskäse, gegrillte Paprika, dazu Oliven und Brot }\end{array}$ \\
\hline $\begin{array}{l}\text { Vier kleine Teufel (Dalmatien Restau- } \\
\text { rant) }\end{array}$ & Cevapcici mit Djuwetschreis und Weißkohl-Salat \\
\hline $\begin{array}{l}\text { Eine Sünde wert (Dalmatien Restau- } \\
\text { rant) }\end{array}$ & $\begin{array}{l}\text { wert Medaillons vom Schweinefilet mit Champi- } \\
\text { gnon- Rahmsauce, dazu Djuwetschreis }\end{array}$ \\
\hline $\begin{array}{l}\text { «Himmlisches Tütchen» (Hotel Bad } \\
\text { Minden) }\end{array}$ & $\begin{array}{l}\text { Fetakäse, Oliven, Paprika, Fleur de Sel und Thy- } \\
\text { mian im Aromabeutelchen gegart }\end{array}$ \\
\hline$\ll$ Teufelsspiejß» (Hotel Bad Minden) & $\begin{array}{l}\text { Seeteufel am Spieß in der Ingwermarinade mit me- } \\
\text { diterranem Gemüse, Safran-Limettenschmand und } \\
\text { Baguettebrotscheiben }\end{array}$ \\
\hline $\begin{array}{l}\text { «Zwischen Himmel und Hölle» (Hotel } \\
\text { Bad Minden) }\end{array}$ & $\begin{array}{l}\text { Frisch aus der Riesenbratpfanne Pfifferlinge mit } \\
\text { Cognac-Kräuter-Rahm und hausgemachten Spätzle }\end{array}$ \\
\hline $\begin{array}{l}\text { «Teuflische Gelüste» (Hotel Bad Min- } \\
\text { den) }\end{array}$ & $\begin{array}{l}\text { Schweinsröllchen gefüllt mit Parmaschinken und } \\
\text { Salbei auf Sugo von Strauchtomaten, dazu Gnocchi }\end{array}$ \\
\hline $\begin{array}{l}\text {... aus vom Himmel gepflückten Oran- } \\
\text { gen und irdischen Karotten (Almundo) }\end{array}$ & Feine Rahmsuppe... \\
\hline $\begin{array}{l}\text { Himmlische Mango... mit Karotten- } \\
\text { stückchen aus dem Erdreich (Al- } \\
\text { mundo) }\end{array}$ & ...und Kokos in einem Topf... \\
\hline
\end{tabular}

Redensarten tritt der Teufel als Widersacher Gottes und als negativer Nachäffer des Göttlichen in Antithese zu Begriffen des Christentums, wie dies auch aus zahlreichen Sagen, Märchen und Sprichwörter bekannt ist, z.B. >Wenn Gott heut' sagt, sagt der Teufel morgen <; > Wo eine Kirche ist, da baut der Teufel eine Kapelle daneben<. http://idiome.deacademic.com/3093/Teufel 


\begin{tabular}{|c|c|}
\hline Name des Gerichtes & Was das ist... \\
\hline „Herrgotts-Bscheißerles“ (Almundo) & $\begin{array}{l}\text { "Gebratene, handgemachte Maultaschen (von den } \\
\text { Schwaben so genannt, weil der Himmel an den an } \\
\text { sich „fleischfreien“ Tagen das im Teig versteckte } \\
\text { Gehackte nicht sehen kann) und schwäbischer Erd- } \\
\text { apfelsalat }\end{array}$ \\
\hline $\begin{array}{l}\text { „Höllenfeuer“ (Hotel \& Restaurant } \\
\text { Große Klus) }\end{array}$ & $\begin{array}{l}\text { Entrecote von der Flammwand mit feuriger Salsa } \\
\text { auf Dicken Bohnen }\end{array}$ \\
\hline $\begin{array}{l}\text { „Himmelsstürmer“ (Hotel \& Restaurant } \\
\text { Große Klus) }\end{array}$ & $\begin{array}{l}\text { Schweinefilet in Schwammerlsoße mit Servietten- } \\
\text { knödel }\end{array}$ \\
\hline $\begin{array}{l}\text { Teuflisch gute Rindersteaks (Bau- } \\
\text { erngut) }\end{array}$ & $\begin{array}{l}\text { Frisch auf der Höllenglut gegrillt - Mit Speckboh- } \\
\text { nen, dazu knuspriges Baguette und Kräuterbutter } \\
\text { Höllisch heißer Genuss! }\end{array}$ \\
\hline $\begin{array}{l}\text { Engelszarte Lammlachse Himmlisches } \\
\text { Rendezvous Verleiht Engelsflügel! } \\
\text { (Bauerngut) }\end{array}$ & von Speckbohnen und Baguette dazu Kräuterbutter. \\
\hline $\begin{array}{l}\text { Himmlische Schweinerückensteaks .... } \\
\text { Direkt vom Fegefeuer. Eine zarte Versu- } \\
\text { chung... Himmlisch schlemmen! Oder: } \\
\text { Heiligenschein garantiert! } \\
\text { (Bauerngut) }\end{array}$ & ...mit Speckbohnen, Baguette und Kräuterbutter. \\
\hline
\end{tabular}

In der biblischen Geschichte der Linsensuppe ${ }^{90}$, kann Esau dem bescheidenen Linsengericht nicht widerstehen. Es geht nicht nur um Gier und Betrug, sondern auch um Verführung und Manipulation, um die Ausnutzung der menschlichen Schwächen, durch Angebote, die zum rechten Zeitpunkt, am rechten Ort stehen und auf die Zielgruppe unwiderstehlich verführerisch wirken. So wird man überzeugt, für das zu zahlen, was man nicht entbehren kann. So wird Esau manipuliert, für die Linsensuppe mit seinem Erstgeburtsrecht zu zahlen. Jedenfalls scheint es ihm geschmeckt zu haben. Er isst und trinkt, steht auf und geht davon - ein Bauchmensch. ${ }^{91}$ Albert Lortzing (18011851) greift dieses Thema in seinem Singspiel Der Waffenschmied humoristisch auf: Die Dummheit bietet selten Zinsen, / sonst leistete ja Esau nicht / für eine Schüssel dicker Linsen / auf seine Erstgeburt Verzicht. ${ }^{92}$

9029 Einst hatte Jakob ein Gericht zubereitet, als Esau erschöpft vom Feld kam. 30 Da sagte Esau zu Jakob: Gib mir doch etwas zu essen von dem Roten, von dem Roten da, ich bin ganz erschöpft. [...]. 31 Jakob gab zur Antwort: Dann verkauf mir jetzt sofort dein Erstgeburtsrecht! 32 Schau, ich sterbe vor Hunger, sagte Esau, was soll mir da das Erstgeburtsrecht? 33 Jakob erwiderte: Schwör mir jetzt sofort! Da schwor er ihm und verkaufte sein Erstgeburtsrecht an Jakob. 34 Darauf gab Jakob dem Esau Brot und Linsengemüse; er aß und trank, stand auf und ging seines Weges. Vom Erstgeburtsrecht aber hielt Esau nichts. (1.Mose 25,29-34) https://www.bibleserver.com/text/EU/1.Mose25

91 http://www.die-bibel.ch/fileadmin/user_upload/PDF/Bibel/Bibelsonntag2016/ Rezept_Linsensuppe.pdf

92 Opernfüher The virtual operahouse Der Waffenschmied Libretto / ERSTER AUFZUG, Nr.2, Arie http://www.opera-guide.ch/opera.php?uilang=de\&id=200\#libretto 


\section{Zum Schluss}

„Erstimhistorischen Rückblickbezeichnen wirdenüberKommunikationssperren laufenden Funktionskreis als Religion und den der Codierung von gutem und schlechtem Verhalten als Moral." Luhmann warnt davor, „zu viel heutigen Sinn über diese Bezeichnungen in Gesellschaften zurückzuprojizieren, deren Kommunikationsweise ganz anders als die unsrige geordnet war. ${ }^{\text {"93 }}$ Das Individuum ist auf sich selbst gestellt, auf sein eigenes Überzeugtsein, er wird nicht gezwungen aus der Sicht irgendeiner Moral oder Religion zu handeln, sondern nur aus Selbstmotivation.

Weder Sprache, noch Religion und Moral oder Recht als Subsysteme der Gesellschaft als Kommunikationssystem können auf die gezielt erfolgs- und gewinnorientierten Handlungen der Menschen, im Geiste von individuellen und GruppenInteressen, einen gründlichen Einfluss nehmen, sie können sie nur spärlich regeln.

\section{Literaturverzeichnis}

Ankenbauer, Norbert. 2010. „Das ich mochte meer newer dyng erfaren“: die Versprachlichung des Neuen in den Paesi novamente retrovati (Vicenza, 1507) und in ihrer deutschen Übersetzung (Nürnberg, 1508). Frank \& Timme GmbH.

Beck, Klaus. 2013. Lasswell-Formel. In Lexikon Kommunikations- und Medienwissenschaft. 2. Auflage, Günter Bentele, Hans-Bernd Brosius, Otfried Jarren (Hrsg.), 182. Wiesbaden: Springer Fachmedien.

Bellmann, Günter. 1989. Zur Nomination und zur Nominationsforschung. Beiträge zur Erforschung der deutschen Sprache 9: 28-31.

Bellmann, Günter. 1996. Der Beitritt als Wende. Referenz und Nomination. In Stand und Aufgaben der deutschen Dialektlexikographie. II. Brüder-Grimm-Symposium zur Historischen Wortforschung. Beiträge zu der Marburger Tagung vom Oktober 1992, Ernst Brenner, Reiner Hildebrandt (Hrsg.), 1-16. Berlin/New York: Walter de Gruyter.

Fleischer, Wolfgang. 1989. Nomination und unfeste nominative Ketten. In Beiträge zur Erforschung der deutschen Sprache, Band 9, Wolfgang Fleischer, Rudolf Große, Gotthard Lerchner (Hrsg.), 13-27. Leipzig.

Fleischer,Wolfgang, Irmhild Barz. 1995. Wortbildung der deutschen Gegenwartssprache. Tübingen: Max Niemeyer Verlag.

Fleischer, Wolfgang, Gerhard Helbig, Gotthard Lerchner. 2001. Kleine Enzyklopädie - Deutsche Sprache. Frankfurt/M.: Peter Lang.

Forcher, Michael, Hans Karl Peterlini. 2010. Südtirol in Geschichte und Gegenwart. Haymon Verlag.

Glück, Helmut, Michael Rödel (Hrsg.). 2016. Metzler Lexikon Sprache. Springer Verlag.

Grauel, Jonas. 2013. Gesundheit, Genuss und gutes Gewissen: Über Lebensmittelkonsum und Alltagsmoral. Transcript Verlag Bielefeld.

Herbermann, Clemens-Peter. 1998. Benennungsprinzipien und Benennungssituationen. $\mathrm{Zu}$ einigen Grundbegriffen der Etymologie. In Lingua Germanica. Studien zur deutschen Philologie. Jochen Splett zum 60. Geburtstag, Eva Schmitsdorf, Nina Hartl, Barbara Meurer (Hrsg.), 70-91. Berlin [u.a.]: Waxmann.

93 Luhmann, 1997, 113. 
Krieg, Ulrike. 2005. Wortbildungsstrategien in der Werbung: zur Funktion und Struktur von Wortneubildungen in Printanzeigen. Hamburg: Buske Verlag.

Luhmann, Niklas. 1997. Die Gesellschaft der Gesellschaft. Frankfurt a. M.: Suhrkamp.

Merten, Klaus. 1977. Kommunikation. Eine Begiffs- und Prozeßanalyse. Opladen.

Nutzinger, Hans G. 1991. Das System der natürlichen Freiheit bei Adam Smith und seine ethischen Grundlagen In Jahrbuch Ökonomie und Gesellschaft, Jahrbuch 9 Adam Smiths Beitrag zur Gesellschaftswissenschaft, 79-100. FrankfurtIM. - New York: Campus.

Pflanz, Florian. 2017. Marketing und Religion. Eine theoretische Untersuchung. Diplomica Verlag.

Platon. [1940]. Sämtliche Werke. Band 1, 541-617. Berlin. Der Text folgt der Übersetzung von Julius Deuschle von 1855 (Permalink: http://www.zeno.org/nid/20009262520). Lizenz: Gemeinfrei Kategorien: Attische Philosophie.

Stewart, George R. 1975. Names on the Globe. New York: Oxford University Press.

Teuteberg, Hans-Jürgen, Günther Wiegelmann. 1986. Unsere tägliche Kost. Geschichte und regionale Prägung. Münster.

Tropp, Jörg. 2014. Moderne Marketing-Kommunikation: System - Prozess - Management. Springer-Verlag.

Turska, Marta. 2009. Internationalismen in der Fachsprache der Gastronomie und Kochkunst im fünfsprachigen Vergleich. Peter Lang.

Wiegand, Herbert Ernst. 1996. Über usuelle und nichtusuelle Benennungskontexte in Alltag und Wissenschaft. In Nomination - fachsprachlich und gemeinsprachlich, Clemens Knobloch Burkhard Schader, 55-103. Opladen: Westdeutscher Verlag.

Internetquellen (alle Internetseiten wurden 2017 abgerufen)

Die Bibel nach Martin Luthers Übersetzung, revidiert 2017, (C) 2016 Deutsche Bibelgesellschaft, Stuttgart https://www.bibleserver.com/text/LUT/Matth\%C3\%A4us4

Düringsfeld, Ida von, Otto Freiherr von Reinsberg-Düringsfeld. 1872. Sprichwörter der germanischen und romanischen Sprachen vergleichend zusammengestellt von Leipzig. Verlag voon Hermann Fries. http://www.bauernregeln.net/kirche.html; http://www.bauernregeln.net/ essen.html

Gabler Wirtschaftslexikon. http://wirtschaftslexikon.gabler.de/Definition/veredelung. html\#definition

Gourmetmeile Minden - auch Veggies werden satt, irgendwie... : Veröffentlicht am 12. Juli 2011. https://missglueckt.wordpress.com/2011/07/12/gourmetmeile-minden-veggie/

Kunst- und Kulturwissenschaft online www.kukwiso.com/index.php/de/nahrungsforschung

Quellenangabe: http://www.minden-erleben.de - Minden Marketing GmbH.

Roland Kühl- v. Puttkamer. Werbung ist von Gott erfunden. http://www.werbeblogger. de/2008/04/04/werbung-ist-von-gott-erfunden/

Smith, Adam. 1904. An Inquiry into the Nature and Causes of the Wealth of Nations. Edwin Cannan (ed.). London: Methuen \& Co., Ltd. 1904. Library of Economics and Liberty [Online]. http://www.econlib.org/library/Smith/smWN1.html (accessed November 22, 2017).

http://www.opera-guide.ch/opera.php?uilang=de\&id=200\#libretto

http://idiome.deacademic.com

www.duden.de

https://www.aphorismen.de

http://dex.dictoo.eu

http://pomologie.com/poire/ 
https://www.namestorm.de/namensagentur

www.poetus.de

http://www.investopedia.com/terms/m/marketing.asp

www.chefkoch.de

https://groups.google.com

http://infozone.forumhit.ro/t364-denumirea-stintifica-a-plantelor

http://www.zeno.org/

www.textatelier.com

https://ro.pinterest.com

http://www.csid.ro/plante-medicinale-fitoterapice-si-gemoterapice/ 